\title{
Inflationary trispectrum from graviton exchange
}

\author{
David Seery ${ }^{1}$, Martin S. Sloth ${ }^{2}$, Filippo Vernizzi ${ }^{3,4}$ \\ ${ }^{1}$ Department of Applied Mathematics and Theoretical Physics, \\ Wilberforce Road, Cambridge, CB3 0WA, United Kingdom \\ ${ }^{2}$ Department of Physics and Astronomy, University of Aarhus \\ Ny Munkegade, DK-8000 Aarhus C, Denmark \\ ${ }^{3} \mathrm{CEA}$, IPhT, 91191 Gif-sur-Yvette cédex, Franceł \\ CNRS, URA-2306, 91191 Gif-sur-Yvette cédex, France \\ ${ }^{4}$ Abdus Salam ICTP, Strada Costiera 11, 34014 Trieste, Italy \\ E-mail: djs61@cam.ac.uk, sloth@phys.au.dk, filippo.vernizzi@cea.fr
}

\begin{abstract}
We compute the connected four-point correlation function of the primordial curvature perturbation generated during inflation with standard kinetic terms, where the correlation is established via exchange of a graviton between two pairs of scalar fluctuations. Any such correlation yields a contribution to the scalar trispectrum of the order of the tensor to scalar ratio $r$. This contribution is numerically one order of magnitude larger than the one previously calculated on the basis of scalar perturbations interacting at a point and satisfies a simple relation in the limit where the momentum of the graviton which is exchanged becomes much smaller than the external momenta. We conclude that the total non-linearity parameter generated by single-field models of slow-roll inflation is at maximum $\left|\tau_{\mathrm{NL}}\right| \sim r$.
\end{abstract}




\section{Introduction}

Since the release of the first-year Wilkinson Microwave Anisotropy Probe (WMAP) satellite data, the availability of high-resolution all-sky maps of the Cosmic Microwave Background (CMB) anisotropy has revolutionized cosmology as a quantitative science. Together with large galaxy surveys, the ever-improving accuracy of the microwave background data has yielded a great deal of precise information concerning conditions in the very early universe. Over the next several years a new generation of CMB instruments will return yet more data, which will fix the parameters of the standard model with even greater precision and may point the way to the discovery of new physics.

Despite its role as the dominant carrier of information from the earliest times, the statistical properties of the CMB temperature anisotropy are remarkably simple: it is known to be statistically Gaussian to very high precision, greater than $0.1 \%$. In recent years, however, the possibility that small non-Gaussian features might be present in these fluctuations has received increasing interest from both cosmologists and particle physicists. This is because primordial non-Gaussianities are one of our best probes of whatever complex physics was operating in the very early universe. In particular, non-Gaussian features discriminate sharply between the minimal implementation of inflation - driven by a single scalar field rolling down its potential under the influence of Hubble friction, known as "slow-roll" dynamics - and more complicated variants involving extra fields or dynamics of greater subtlety.

Which observables are relevant in the study of non-Gaussianity? An exactly Gaussian process is one in which all statistical information is encoded in the two-point expectation value. Any observation of new information in the three- or higher $n$-point correlation functions therefore constitutes an observation of non-Gaussianity. Other statistical measures exist, such as Minkowski functionals [1]. However, at the present day the best confrontation of theory with observation is by means of the three-point correlation function of the curvature perturbation, $\zeta$.

To make this quantitative, one defines the bispectrum $B_{\zeta}$ by

$$
\left\langle\zeta\left(\mathbf{k}_{1}\right) \zeta\left(\mathbf{k}_{2}\right) \zeta\left(\mathbf{k}_{3}\right)\right\rangle \equiv(2 \pi)^{3} \delta\left(\sum_{a} \mathbf{k}_{a}\right) B_{\zeta}\left(\mathbf{k}_{1}, \mathbf{k}_{2}, \mathbf{k}_{3}\right),
$$

where $B_{\zeta}$ is a function of the triangle formed by the three momenta $\left\{\mathbf{k}_{1}, \mathbf{k}_{2}, \mathbf{k}_{3}\right\}$, and we have introduced a notation which will be used throughout this paper, in which Latin indices such as $a, b, c, \ldots$ range from 1 to the number of momenta carried by the correlation function in question. These are to be distinguished from Latin indices such as $i, j, l, \ldots$, ranging from 1 to 3 which label the spatial dimensions. It is customary to introduce a dimensionless quantity, $f_{\mathrm{NL}}$, which generally depends on the momenta $k_{a}$, and parametrizes the strength of the non-Gaussian signal. We define

$$
B_{\zeta} \equiv \frac{6}{5} f_{\mathrm{NL}} \sum_{a<b} P_{\zeta}\left(k_{a}\right) P_{\zeta}\left(k_{b}\right),
$$

where $P_{\zeta}\left(k_{1}\right)$ is the power spectrum of $\zeta$, such that $\left\langle\zeta\left(\mathbf{k}_{1}\right) \zeta\left(\mathbf{k}_{2}\right)\right\rangle=(2 \pi)^{3} \delta\left(\sum_{a} \mathbf{k}_{a}\right) P_{\zeta}\left(k_{1}\right)$. Occasionally, $f_{\mathrm{NL}}$ is referred to as the non-linearity parameter $[2,3]$. An $f_{\mathrm{NL}}$ that is 
independent of the momenta corresponds to a local type of non-Gaussianity, which arises from the real space relation

$$
\zeta=\zeta_{g}+\frac{3}{5} f_{\mathrm{NL}}^{\text {local }}\left(\zeta_{g}^{2}-\left\langle\zeta_{g}^{2}\right\rangle\right)
$$

where $\zeta_{g}$ is Gaussian. In this case, the bispectrum is maximized in the limit of one of the three momenta going to zero [4]. This form of non-Gaussianity is produced by nonlinear gravitational evolution subsequent to horizon crossing $[4,5]$.

On the basis of non-linear perturbation theory we might naïvely expect a nonlinearity parameter of order unity, i.e., $f_{\mathrm{NL}} \sim \mathrm{O}(1)$. However (neglecting post-processing in the later universe from the ubiquitous non-linearities of gravity $[6,7,8,9,10]$ ) in single-field slow-roll inflation $f_{\mathrm{NL}}$ is very small-in fact, it is of the same order as the slow-roll parameters $[11,12]$, which are required to be much smaller than unity. Such a small non-linearity is beyond our present experimental reach, which is $\left|f_{\mathrm{NL}}\right| \gtrsim 100$. However, in the near future the Planck satellite may be sensitive to $f_{\mathrm{NL}}$ of order unity [13]. Any detection of a primordial $\left|f_{\mathrm{NL}}\right|$ larger than order unity will strongly disfavour minimal inflation.

It is possible that interesting information might be encoded in the higher-order $n$ point functions, such as the connected part of the four-point function [14]. Constraining such an expectation value from CMB data is extremely challenging and at the time of writing no such analysis has been completed for the WMAP data, although a study on small angular scales has been carried out for the BOOMERanG data [15] and an all-sky analysis of the four-point function exists for the older COBE satellite [16]. However, as computing power increases and algorithms become more efficient, it is quite plausible that it will be possible to extract four-point correlations for the WMAP data, and that this will be possible for Planck. In view of future constraints from CMB experiments and elsewhere (see for instance Refs. $[17,18]$ ), the purpose of this paper is to clarify the predictions of single-field slow-roll models of inflation for the four-point correlation function of the curvature perturbation $\zeta$.

The four-point correlation function is conventionally parametrized in terms of the trispectrum, written $T_{\zeta}$, and defined by $[19,20]$

$$
\left\langle\zeta\left(\mathbf{k}_{1}\right) \zeta\left(\mathbf{k}_{2}\right) \zeta\left(\mathbf{k}_{3}\right) \zeta\left(\mathbf{k}_{4}\right)\right\rangle=(2 \pi)^{3} \delta\left(\sum_{a} \mathbf{k}_{a}\right) T_{\zeta}\left(\mathbf{k}_{1}, \mathbf{k}_{2}, \mathbf{k}_{3}, \mathbf{k}_{4}\right),
$$

where by convention only the connected part of the expectation value on the left-hand side is considered. The disconnected part is present even in an exactly Gaussian model, and forms a background from which the genuinely non-linear signal must be extracted. Unlike the bispectrum, the trispectrum typically depends on the relative orientations of the $\mathbf{k}_{a}$ in addition to their magnitudes. If observation forces us to abandon the minimal scenario of inflation, then the trispectrum is a ready-made observable which could be consulted for guidance concerning the modifications which are required.

The trispectrum can be parametrized as [21]

$$
T_{\zeta} \equiv \tau_{\mathrm{NL}} \sum_{\substack{b<c \\ a \neq b, c}} P_{\zeta}\left(k_{a b}\right) P_{\zeta}\left(k_{b}\right) P_{\zeta}\left(k_{c}\right)+\frac{54}{25} g_{\mathrm{NL}} \sum_{a<b<c} P_{\zeta}\left(k_{a}\right) P_{\zeta}\left(k_{b}\right) P_{\zeta}\left(k_{c}\right)
$$




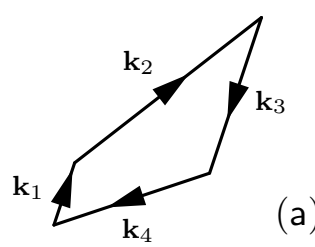

(a)

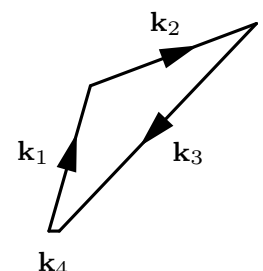

(b)

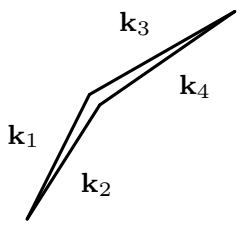

(c)

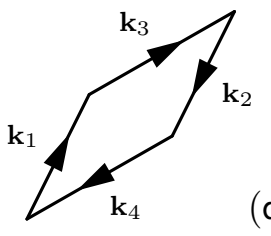

(d)

Figure 1. Possible planar momentum quadrilaterals. Note, however, that there is no need for the quadrilateral to lie in a plane, and in general it is a three-dimensional object. In (a), there is no particular relationship among the sides of the quadrilateral. This is the general case. In (b), the side associated with $\mathbf{k}_{4}$ is taken to zero length, causing the quadrilateral to degenerate to a triangle. In (c), adjacent sides possess equal magnitudes and their directions are becoming opposite, a limit we refer to as the folded kite. In (d), opposite sides possess equal magnitudes, yielding a parallelogram. The shapes described in (c) and (d), are therefore precisely dual to each other, and describe the same physics.

where $k_{a b} \equiv\left|\mathbf{k}_{a b}\right|$ and $\mathbf{k}_{a b} \equiv \mathbf{k}_{a}+\mathbf{k}_{b}$. This definition is motivated by the fact that, for $\tau_{\mathrm{NL}}$ and $g_{\mathrm{NL}}$ which are independent of the momenta, this is the most general local parametrization, corresponding to a non-Gaussianity of the form

$$
\zeta=\zeta_{g}+\frac{1}{2}\left(\tau_{\mathrm{NL}}^{\text {local }}\right)^{1 / 2}\left(\zeta_{g}^{2}-\left\langle\zeta_{g}^{2}\right\rangle\right)+\frac{9}{25} g_{\mathrm{NL}}^{\text {local }} \zeta_{g}^{3}
$$

in real space. As for the bispectrum, this simple momentum dependence characterized by constant $\tau_{\mathrm{NL}}^{\text {local }}$ and $g_{\mathrm{NL}}^{\text {local }}$ is generically produced by gravitational evolution subsequent to horizon crossing.

More generally, the classical gravitational evolution generates a curvature perturbation which can be expanded in powers of $\zeta_{g}$ with definite momentumindependent coefficients. In such a case, if the perturbations are generated by only one field, one expects that [21]

$$
\tau_{\mathrm{NL}}^{\text {local }}=\left(\frac{6}{5} f_{\mathrm{NL}}^{\text {local }}\right)^{2} .
$$

However, the curvature perturbation during inflation is subject to quantum interference effects around the time of horizon crossing, and does not admit a simple parameterization in terms of momentum independent-coefficients [22, 4]. It follows that in general there need be $n o$ relationship between $\tau_{\mathrm{NL}}$ and $f_{\mathrm{NL}}$.

There are two interesting degenerate configurations where a non-Gaussianity of local form maximizes the trispectrum. The first is the so-called "squeezed" limit, where one of the four momenta is taken to zero. In this limit, the quadrilateral formed by the momentum vectors $\mathbf{k}_{a}$ degenerates into a triangle. (See Fig. 1(a)-(b).) For instance, by taking $k_{4} \ll k_{1}, k_{2}, k_{3}$ one obtains

$T_{\zeta}^{\text {local }}=\left(2 \tau_{\mathrm{NL}}^{\text {local }}+\frac{54}{25} g_{\mathrm{NL}}^{\text {local }}\right) P_{\zeta}\left(k_{4}\right)\left[P_{\zeta}\left(k_{1}\right) P_{\zeta}\left(k_{2}\right)+P_{\zeta}\left(k_{1}\right) P_{\zeta}\left(k_{3}\right)+P_{\zeta}\left(k_{2}\right) P_{\zeta}\left(k_{3}\right)\right]$.

Thus, $\tau_{\mathrm{NL}}^{\text {local }}$ and $g_{\mathrm{NL}}^{\text {local }}$ contribute equally to the trispectrum in this limit. 
The second interesting limit occurs when the magnitude of the sum of two momenta is taken to zero, so that $k_{i j}=\left|\mathbf{k}_{i}+\mathbf{k}_{j}\right| \rightarrow 0$ for some $i \neq j$. We refer to this limit as the counter-collinear case because for each momentum there is another one with equal magnitude and opposite direction. One can visualize the quadrilateral formed by the momentum vectors in this limit in two different but equivalent ways. If we choose to order the momenta so that the counter-collinear pairs are adjacent, then we generate what can be called a "folded kite," shown in Fig. 1(c). On the other hand, if we choose to order the momenta so that the counter-collinear pairs are on opposite sides of the quadrilateral, then it becomes planar and yields a parallelogram, as in Fig. 1(d). Changing the order of the momenta does not affect the trispectrum, which is a function only of the intrinsic geometry of the $\mathbf{k}_{a}$; for the trispectrum, this means it depends on a total of three magnitudes and three angles. Therefore, the planar shapes given in Fig. 1(c)-(d) are precisely dual to each other. The local trispectrum simplifies in the counter-collinear limit. By taking $k_{12} \ll k_{1} \approx k_{2}, k_{3} \approx k_{4}$ one obtains

$$
T_{\zeta}^{\text {local }}\left(\mathbf{k}_{1}, \mathbf{k}_{2}, \mathbf{k}_{3}, \mathbf{k}_{4}\right)=4 \tau_{\mathrm{NL}}^{\text {local }} P_{\zeta}\left(k_{12}\right) P_{\zeta}\left(k_{1}\right) P_{\zeta}\left(k_{3}\right) \text {. }
$$

Thus, only $\tau_{\mathrm{NL}}^{\text {local }}$ contributes to the trispectrum in this limit.

As discussed above, it does not always happen that a model of the early universe predicts a curvature perturbation whose non-linearities are precisely of the local form described by Eqs. (1.3) and (1.6). To accommodate this situation, one can take $\tau_{\mathrm{NL}}$ and $g_{\mathrm{NL}}$ as momentum dependent; however, in this case the separation between $\tau_{\mathrm{NL}}$ and $g_{\mathrm{NL}}$ becomes ambiguous. Indeed, several authors $[23,5,24]$ have used a momentum dependent $\tau_{\mathrm{NL}}$ alone to parametrize the full trispectrum. The division of the trispectrum into terms proportional to $\tau_{\mathrm{NL}}$ and $g_{\mathrm{NL}}$ is helpful where these parameters can be made approximately momentum-independent, but this is not always the case when the nonGaussianity is dominated by quantum interference effects around the time of horizon exit, as in single-field inflation. For this reason we will mostly avoid the use of $\tau_{\mathrm{NL}}$ or $g_{\mathrm{NL}}$ in this paper, giving expressions for the trispectrum directly. However, as we will see, the contribution of graviton exchange to the trispectrum is dominant in the parallelogram limit, in such a way that we can describe it as a momentum independent $\tau_{\mathrm{NL}}^{\text {local }}$.

In perturbation theory our expectation would again be $\tau_{\mathrm{NL}} \sim g_{\mathrm{NL}}=\mathrm{O}(1)$. However, it has been shown that, in slow-roll inflation with canonical kinetic term, $\S \tau_{\mathrm{NL}}$ exhibits a similar suppression by one power of the slow-roll parameter $\epsilon \equiv-\dot{H} / H^{2}$, giving $\tau_{\mathrm{NL}} \lesssim r / 50$ [28], where $r$ is the ratio of tensor to scalar amplitudes, $r \equiv 8 P_{T} / P_{\zeta}$,

$\S$ The calculation of the trispectrum in single-field models with non-canonical kinetic terms was first performed, neglecting metric perturbations, in Ref. [26] and later, more generally, in Ref. [27]. In these models $\tau_{\mathrm{NL}}$ can be of order unity or larger. The contribution from the graviton exchange is suppressed by slow-roll with respect to the one due to the contact interaction and it has been neglected in those references. Note that the calculation performed in this paper, i.e., of the trispectrum from slow-roll inflation models based on a field with standard kinetic term, requires one to pursue the calculation to one order higher in slow-roll than was done in Refs. [26, 27]. Thus, those references do not include our final results. 

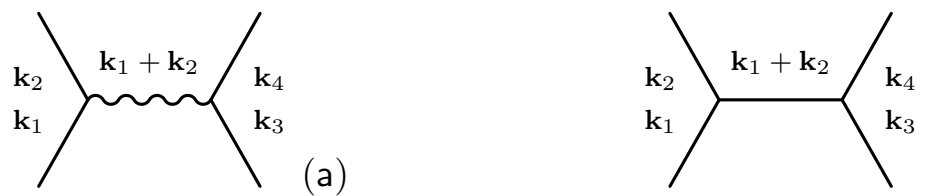

Figure 2. In (a), exchange of a graviton (represented by a wavy line) leads to correlations among four scalar fluctuations (represented by straight lines). In the limit where the graviton which is exchanged becomes extremely soft, so that $\left|\mathbf{k}_{1}+\mathbf{k}_{2}\right| \rightarrow 0$, one can think of such correlations as being mediated by fluctuations on top of a modified background which carries a classical gravitational wave. The same interpretation holds in (b), where the exchange is mediated by a scalar particle.

and in single-field inflation $r=16 \epsilon$. It follows that the non-linearity parameter $\tau_{\mathrm{NL}}$ is roughly of the same size as $f_{\mathrm{NL}}$. (In practice, of course, the trispectrum is much harder to detect than the bispectrum because an extra power of the power spectrum $P_{\zeta}$ is equivalent to suppression by $\sim 10^{-10}$.) However, one may have some reservations about this conclusion. In particular, the calculation reported in Ref. [28] ignored nonlinear interactions mediated by scalar or tensor fluctuations. In terms of diagrams, this is equivalent to neglecting the two diagrams where the correlation among the four scalar modes is due to the exchange of scalar or tensor quanta. The latter diagram, which turns out to be more important, is shown in Fig. (2)(a), whereas the scalar exchange is described in Fig. 2(b).

The vertices of these nonlinear interaction can be computed straightforwardly using the action for Einstein gravity coupled to a single scalar field, which was studied to third order by Maldacena [11]. For the vertex which describes a contact interaction between three quanta of the curvature perturbation, $\zeta$, the appropriate term in the Lagrangian is typically suppressed by at least one power of the slow-roll parameters, i.e., $\mathcal{L}_{\zeta \zeta \zeta} / \mathcal{L}_{\zeta \zeta} \sim \mathrm{O}(\epsilon, \eta) \cdot P_{\zeta}^{1 / 2}$ and therefore

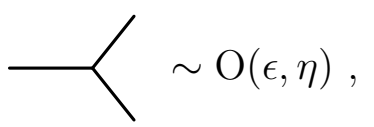

where $\eta$ is the second slow-roll parameter, defined as $\eta \equiv M_{\mathrm{P}}^{2} V^{\prime \prime} / V$, where we have introduced the reduced Planck mass $M_{\mathrm{P}} \equiv(8 \pi G)^{-1 / 2}$. Thus, bearing in mind that a four-point correlation established by scalar exchange must contain two vertices of this sort, we expect that the contribution to the trispectrum from this process is of the order of two powers of slow-roll, i.e., $\tau_{\mathrm{NL}} \sim \mathrm{O}\left(\epsilon^{2}, \eta^{2}, \epsilon \eta\right)$. This is suppressed by one power of slow-roll compared with the scalar contact interaction and can therefore be ignored. However, things are different when we consider the exchange of a graviton. In this case the interaction Lagrangian between a graviton and two scalars is not suppressed by any powers of slow-roll parameters at all, i.e., $\mathcal{L}_{\gamma \zeta \zeta} / \mathcal{L}_{\zeta \zeta} \sim P_{\gamma}^{1 / 2}$ and therefore

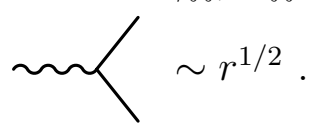

The power spectrum of gravitational waves is smaller than that of $\zeta$ by a factor of $r$, and therefore this contribution is of order $r$. It follows that the effect of graviton exchange is 
comparable to the contact contribution studied in Ref. [28]. This observation was first made by Arroja \& Koyama [27].॥

In the present paper, we will calculate the contribution to the four-point function of scalar curvature perturbations which arises from correlations mediated by graviton exchange. We explicitly carry out this calculation for single-field models, but our result is easily generalized to many fields and our final results will apply to multifield examples. Like many previous calculations of the non-linearities among scalar fluctuations at horizon crossing, this calculation is somewhat involved. Fortunately, in the counter-collinear limit (i.e., parallelogram or folded-kite), the amplitude and momentum dependence of the contribution to the trispectrum from graviton exchange can be computed straightforwardly by a simple semiclassical argument, which provides an important check of our computation.

In the next section we explicitly compute the contribution to the trispectrum from graviton exchange, using the uniform curvature gauge, while in $\S 3$ we discuss the counter-collinear limit and check our calculation. In $\S 4$ we give an expression for the total trispectrum, including the contribution of Ref. [28] and in $§ 5$ we conclude with a discussion. Finally, in Appendix A we report the calculation of a long double integral used in $\S 2$.

Throughout this paper, we use fundamental units in which Planck's constant $\hbar$ and the speed of light $c$ are set to unity, so $\hbar=c=1$. We choose to set $M_{\mathrm{P}}^{-2} \equiv 8 \pi G$ equal to unity. Our metric convention is $(-,+,+,+)$.

\section{Trispectrum from graviton exchange}

In this section we want to evaluate the contribution to the four-point function of curvature perturbations from the exchange of a graviton. This is the process shown in Fig. 2(a), which involves a third-order interaction among scalar fluctuations and tensor perturbations of the metric. The description of this interaction requires that we expand the action to third order in such fluctuations. For simplicity, we will restrict to a single scalar field only. The calculation can easily be extended to more than one field, and indeed our final result for the trispectrum of the curvature perturbation $\zeta$ remains valid in the multi-field case with flat field space metric.

We begin with Einstein gravity coupled to a scalar field, which gives a total action

$$
S=\frac{1}{2} \int \sqrt{-g}\left[R-(\nabla \phi)^{2}-2 V(\phi)\right]
$$

|| In Ref. [27] it was also claimed that the discussion of Ref. [29] neglected tensor modes. However, the tensor contribution originally derived by Maldacena [11], which was discussed again in Ref. [27], was in fact included in Ref. [29], where it was shown to confirm their conclusions.

One might also worry that the graviton-scalar-scalar vertex could dominate corrections to the scalar power spectrum, which arise via loops which exchange virtual quanta. The correction to the scalar power spectrum due to loops from the graviton-scalar-scalar interaction has been computed in [30], where it was shown that it is of the same magnitude as that coming from scalar self-interactions $[31,32,33,34,35]$. 
where $R$ is the spacetime Ricci scalar and $V(\phi)$ is an arbitrary potential. Using the Arnowitt-Deser-Misner method (or so-called "ADM formalism") [36], one can derive the action for coupled scalar and perturbations from Eq. (2.1) to any given order using an iterative procedure. The ADM line element is given by

$$
d s^{2}=-N^{2} d t^{2}+a^{2}(t) h_{i j}\left(d x^{i}+N^{i} d t\right)\left(d x^{j}+N^{j} d t\right)
$$

where $a(t)$ is the scale factor, and $N, N^{i}$ are the lapse and the shift functions. The threedimensional metric $h_{i j}$ encodes scalar and tensor fluctuations in the spatial geometry. When the ADM metric ansatz is inserted into Eq. (2.1), the lapse and the shift functions act as Lagrange multipliers: the field equations obtained by minimizing the action in $N$ and $N^{i}$ give the constraint equations which follow from the Einstein equation, while $\phi$ and $h_{i j}$ are the dynamical degrees of freedom.

As in Ref. [28], we choose to work in the uniform curvature gauge. In this gauge we take the scalar field and the spatial metric to be parametrized by

$$
\phi=\phi(t)+\varphi(t, \mathbf{x}), \quad h_{i j}=a(t)\left(e^{\gamma}\right)_{i j} .
$$

Here $\varphi(t, \mathbf{x})$ is the inflaton field fluctuation, and $\gamma_{i j}$ is a traceless and divergenceless tensor fluctuation, obeying $\partial_{i} \gamma_{i j}=\gamma_{i i}=0$. Our eventual aim is to compute the four-point function of the comoving curvature perturbation, $\mathcal{R}$, which on super-Hubble scales is equal to the curvature perturbation on uniform density slices, $\zeta[37,38,39]$. However, the calculation is considerably simplified if we choose to calculate the fourpoint function of $\varphi$ at horizon crossing and then relate it to the four-point function of the uniform density curvature perturbation $\zeta$. Indeed, as shown in Ref. [11], in virtue of the smallness of the slow-roll parameters at Hubble crossing, it is possible to reproduce the correct late-time behaviour of $\zeta$ using the free-field solution in de Sitter spacetime for $\varphi$. At late times $\zeta$ is constant and, at lowest order in slow-roll, it can simply be normalized to $\varphi$ at horizon crossing.

The free action, which describes the evolution of independent scalar and tensor fluctuations, is

$S_{2}=\int d^{3} x d t a^{3}\left[\frac{1}{2}\left(\dot{\varphi}^{2}-\frac{\partial_{i} \varphi \partial_{i} \varphi}{a^{2}}\right)+\frac{1}{8}\left(\dot{\gamma}_{i j} \dot{\gamma}_{i j}-\frac{\partial_{k} \gamma_{i j} \partial_{k} \gamma_{i j}}{a^{2}}\right)\right]$

where we are using the convention that a repeated spatial index in the lowered position, such as $i$ or $j$, denotes summation with the flat spatial metric $\delta_{i j}$. The free fields derived from this action satisfy the equations of motion obtained by varying $\varphi$ and $\gamma_{i j}$, and can therefore be built out of mode functions $U_{k}(t), U_{k}^{*}(t)$ and $\gamma_{k}^{s}(t), \gamma_{k}^{s *}(t)$ which solve the classical equations of motion,

$$
\begin{aligned}
U_{k}(\eta) & =\frac{H_{*}}{\sqrt{2 k^{3}}}(1-i k \eta) e^{i k \eta}, \\
\gamma_{k}^{s}(\eta) & =\frac{H_{*}}{\sqrt{k^{3}}}(1-i k \eta) e^{i k \eta} \quad(\text { any } s),
\end{aligned}
$$

where $\eta$ is the conformal time, defined by $d \eta=d t / a$, and $H_{*}$ is the Hubble parameter evaluated at Hubble crossing for the mode $k$; an asterisk "*" as superscript denotes 
complex conjugation. The index $s$ labels the two possible polarization states "+" and " $x$ " of a spin two gravitational excitation. Introducing creation and annihilation operators $a_{\mathbf{k}}^{+}, a_{\mathbf{k}}^{-}$for the scalar quanta we obtain

$$
\varphi(x)=\int \frac{d^{3} k}{(2 \pi)^{3}}\left[a_{\mathbf{k}}^{-} U_{k}(t)+a_{-\mathbf{k}}^{+} U_{k}^{*}(t)\right] e^{i \mathbf{k} \cdot \mathbf{x}},
$$

whereas, with corresponding operators $b_{\mathbf{k}}^{+s}, b_{\mathbf{k}}^{-s}$ for each polarization of the gravitational fluctuation, we find

$$
\gamma_{i j}(x)=\sum_{s=+, \times} \int \frac{d^{3} k}{(2 \pi)^{3}}\left[b_{\mathbf{k}}^{-s} \epsilon_{i j}^{s}(\mathbf{k}) \gamma_{k}^{s}(t)+b_{-\mathbf{k}}^{+s} \epsilon_{i j}^{s}(-\mathbf{k}) \gamma_{k}^{s *}(t)\right] e^{i \mathbf{k} \cdot \mathbf{x}} .
$$

The polarization tensors $\epsilon_{i j}^{s}$ are chosen to satisfy the transversality and tracelessness conditions $\epsilon_{i i}^{s}(\mathbf{k})=k^{i} \epsilon_{i j}^{s}(\mathbf{k})=0$, together with a completeness relation obtained by tracing over spatial indices, $\epsilon_{i j}^{s}(\mathbf{k}) \epsilon_{i j}^{* s^{\prime}}(\mathbf{k})=2 \delta_{s s^{\prime}}$

We have now reduced the problem to calculating the four-point function of fluctuations $\varphi$ in the inflaton field at horizon crossing, where the correlations are produced via the graviton exchange diagram. To proceed, we need only the relevant third order term in the action, which describes the graviton-scalar-scalar vertex in uniform curvature gauge. This is [11],

$$
S_{3}=\frac{1}{2} \int d^{3} x d \eta a^{2} \gamma^{i j} \partial_{i} \varphi \partial_{j} \varphi
$$

The correct approach to computing expectation values in an expanding or curved background spacetime is the so-called in-in or Schwinger-Keldysh formalism [40, 41, 42]. In this formalism the expectation value of any operator $\mathcal{O}$ (evaluated at time $\eta_{*}$ ) is given by

$$
\left\langle\Omega\left|\mathcal{O}\left(\eta_{*}\right)\right| \Omega\right\rangle=\frac{\left\langle 0\left|\mathrm{~T}\left\{\mathcal{O}\left(\eta_{*}\right) e^{-i \int_{-\infty}^{\eta_{*}} d \eta\left[H_{\mathrm{I}}\left(\phi, \varphi^{+}, \gamma^{+}\right)-H_{\mathrm{I}}\left(\phi, \varphi^{-}, \gamma^{-}\right)\right]}\right\}\right| 0\right\rangle}{\left\langle 0\left|\mathrm{~T}\left\{e^{-i \int_{-\infty}^{\eta_{*}} d \eta\left[H_{\mathrm{I}}\left(\phi, \varphi^{+}, \gamma^{+}\right)-H_{\mathrm{I}}\left(\phi, \varphi^{-}, \gamma^{-}\right)\right]}\right\}\right| 0\right\rangle},
$$

where $|\Omega\rangle$ is the vacuum of the interacting theory while $|0\rangle$ is the vacuum of the free theory, $\mathrm{T}$ is the time ordering operator and $H_{\mathrm{I}}$ is the interaction Hamiltonian given, in our case, by 9

$$
H_{\mathrm{I}}=-\frac{1}{2} \int d^{3} x a^{2} \gamma^{i j} \partial_{i} \varphi \partial_{j} \varphi
$$

The matrix element (2.10) describes a system evolved from its initial vacuum state at $\eta \rightarrow-\infty$ to $\eta=\eta_{*}$ with an operator inserted at $\eta$, and back again from $\eta_{*}$ to $-\infty$, with a set of "+" fields on the increasing-time contour and a set of "-" fields on the

I In general, the interaction Hamiltonian does not have to be given by the sign reverse of the interaction Lagrangian. This is only true in a limited class of theories without derivative interactions, which does not include the case of coupled matter and metric fluctuations during inflation. It follows that extra terms can be generated in the transformation to the interaction Hamiltonian [43, 25]. For models with non-minimal kinetic terms, this can lead to non-negligible contributions to the trispectrum, as discussed in v3 of Ref. [26]. In the case of slow-roll inflation with canonical kinetic terms, however, these effects can be ignored. 
decreasing-time contour. Eventually, $\eta_{*}$ will be taken to 0 and the final result is finite in this limit.

The contractions between different pairs of the two types of field perturbations yield four kinds of propagator,

$$
G^{ \pm \pm}\left(x, x^{\prime}\right) \equiv\left\langle 0\left|\mathrm{~T}\left[\varphi^{ \pm}(x) \varphi^{ \pm}\left(x^{\prime}\right)\right]\right| 0\right\rangle .
$$

It is convenient to rewrite this expression in Fourier space, where the propagator takes the form

$$
G^{ \pm \pm}\left(x, x^{\prime}\right)=\int \frac{d^{3} k}{(2 \pi)^{3}} e^{i \mathbf{k} \cdot\left(\mathbf{x}-\mathbf{x}^{\prime}\right)} G_{k}^{ \pm \pm}\left(\eta, \eta^{\prime}\right),
$$

and $G_{k}^{ \pm \pm}$can be written as

$$
\begin{aligned}
& G_{k}^{++}\left(\eta, \eta^{\prime}\right)=G_{k}^{>}\left(\eta, \eta^{\prime}\right) \Theta\left(\eta-\eta^{\prime}\right)+G_{k}^{<}\left(\eta, \eta^{\prime}\right) \Theta\left(\eta^{\prime}-\eta\right), \\
& G_{k}^{--}\left(\eta, \eta^{\prime}\right)=G_{k}^{>}\left(\eta, \eta^{\prime}\right) \Theta\left(\eta^{\prime}-\eta\right)+G_{k}^{<}\left(\eta, \eta^{\prime}\right) \Theta\left(\eta-\eta^{\prime}\right), \\
& G_{k}^{-+}\left(\eta, \eta^{\prime}\right)=G_{k}^{>}\left(\eta, \eta^{\prime}\right) \\
& G_{k}^{+-}\left(\eta, \eta^{\prime}\right)=G_{k}^{<}\left(\eta, \eta^{\prime}\right) .
\end{aligned}
$$

The " $>$ " and " $<$ " functions are defined by

$$
\begin{aligned}
& G_{k}^{>}\left(\eta, \eta^{\prime}\right)=U_{k}(\eta) U_{k}^{*}\left(\eta^{\prime}\right), \\
& G_{k}^{<}\left(\eta, \eta^{\prime}\right)=U_{k}^{*}(\eta) U_{k}\left(\eta^{\prime}\right) .
\end{aligned}
$$

Similarly, for the tensor modes we have a propagator $F_{i j l m}^{s s^{\prime} \pm \pm}$

$$
F_{i j l m}^{s s^{\prime} \pm \pm}\left(x, x^{\prime}\right) \equiv\left\langle 0\left|\mathrm{~T}\left[\gamma_{i j}^{s \pm}(x) \gamma_{l m}^{s^{\prime} \pm}\left(x^{\prime}\right)\right]\right| 0\right\rangle,
$$

which goes over to the Fourier space representation

$$
F_{i j l m}^{s s^{\prime} \pm \pm}\left(x, x^{\prime}\right)=\sum_{s=+, \times} \int \frac{d^{3} k}{(2 \pi)^{3}} e^{i \mathbf{k} \cdot\left(\mathbf{x}-\mathbf{x}^{\prime}\right)} \epsilon_{i j}^{s}(\mathbf{k}) \epsilon_{l m}^{s}(-\mathbf{k}) F_{k}^{s \pm \pm}\left(\eta, \eta^{\prime}\right) .
$$

As before, explicit expressions can be given in terms of " $>$ " and " $<$ " functions, leading to

$$
\begin{aligned}
& F_{k}^{s++}\left(\eta, \eta^{\prime}\right)=F_{k}^{s>}\left(\eta, \eta^{\prime}\right) \Theta\left(\eta-\eta^{\prime}\right)+F_{k}^{s<}\left(\eta, \eta^{\prime}\right) \Theta\left(\eta^{\prime}-\eta\right), \\
& F_{k}^{s--}\left(\eta, \eta^{\prime}\right)=F_{k}^{s>}\left(\eta, \eta^{\prime}\right) \Theta\left(\eta^{\prime}-\eta\right)+F_{k}^{s<}\left(\eta, \eta^{\prime}\right) \Theta\left(\eta-\eta^{\prime}\right), \\
& F_{k}^{s-+}\left(\eta, \eta^{\prime}\right)=F_{k}^{s>}\left(\eta, \eta^{\prime}\right), \\
& F_{k}^{s+-}\left(\eta, \eta^{\prime}\right)=F_{k}^{s<}\left(\eta, \eta^{\prime}\right),
\end{aligned}
$$

where one has

$$
\begin{aligned}
& F_{k}^{s>}\left(\eta, \eta^{\prime}\right)=\gamma_{k}^{s}(\eta) \gamma_{k}^{s *}\left(\eta^{\prime}\right), \\
& F_{k}^{s<}\left(\eta, \eta^{\prime}\right)=\gamma_{k}^{s *}(\eta) \gamma_{k}^{s}\left(\eta^{\prime}\right) .
\end{aligned}
$$

Expanding the exponential in Eq. (2.10) to second order, using the interaction Hamiltonian given by Eq. (2.11), and making all possible Wick contractions among the fields gives the contribution to the four-point function from graviton exchange (which we label with the superscript "GE"). Since there are two vertices in the diagram, the 
result depends on two time integrals. Using Eqs. (2.12) and (2.16) to carry out the Wick contractions, together with the Fourier space definitions (2.13) and (2.17), leads to

$$
\begin{aligned}
\left\langle\varphi_{\mathbf{k}_{1}} \varphi_{\mathbf{k}_{2}} \varphi_{\mathbf{k}_{3}} \varphi_{\mathbf{k}_{4}}\right\rangle_{*}^{\mathrm{GE}} & =-\frac{1}{4}(2 \pi)^{3} \delta^{3}\left(\sum_{a} \mathbf{k}_{a}\right) \sum_{s} \epsilon_{i j}^{s}\left(\mathbf{k}_{12}\right) k_{1}^{i} k_{2}^{j} \epsilon_{l m}^{s}\left(\mathbf{k}_{34}\right) k_{3}^{l} k_{4}^{m} \int_{-\infty}^{\eta_{*}} \frac{d \eta}{\eta^{2}} \int_{-\infty}^{\eta} \frac{d \eta^{\prime}}{\eta^{\prime 2}} \\
\times & {\left[G_{k_{1}}^{++}\left(\eta_{*}, \eta\right) G_{k_{2}}^{++}\left(\eta_{*}, \eta\right) G_{k_{3}}^{++}\left(\eta_{*}, \eta^{\prime}\right) G_{k_{4}}^{++}\left(\eta_{*}, \eta^{\prime}\right) F_{k_{12}}^{s++}\left(\eta, \eta^{\prime}\right)\right.} \\
& +G_{k_{1}}^{+-}\left(\eta_{*}, \eta\right) G_{k_{2}}^{+-}\left(\eta_{*}, \eta\right) G_{k_{3}}^{+-}\left(\eta_{*}, \eta^{\prime}\right) G_{k_{4}}^{+-}\left(\eta_{*}, \eta^{\prime}\right) F_{k_{12}}^{s-}\left(\eta, \eta^{\prime}\right) \\
& -G_{k_{1}}^{++}\left(\eta_{*}, \eta\right) G_{k_{2}}^{++}\left(\eta_{*}, \eta\right) G_{k_{3}}^{+-}\left(\eta_{*}, \eta^{\prime}\right) G_{k_{4}}^{+-}\left(\eta_{*}, \eta^{\prime}\right) F_{k_{12}}^{s+-}\left(\eta, \eta^{\prime}\right) \\
& \left.-G_{k_{1}}^{+-}\left(\eta_{*}, \eta\right) G_{k_{2}}^{+-}\left(\eta_{*}, \eta\right) G_{k_{3}}^{++}\left(\eta_{*}, \eta^{\prime}\right) G_{k_{4}}^{++}\left(\eta_{*}, \eta^{\prime}\right) F_{k_{12}}^{s-+}\left(\eta, \eta^{\prime}\right)\right] \\
+ & 23 \text { permutations }
\end{aligned}
$$

Using $G_{k}^{++}\left(\eta_{*}, \eta\right)=G^{>}\left(\eta_{*}, \eta\right)$ for $\eta_{*}>\eta, F_{k}^{++}\left(\eta, \eta^{\prime}\right)=F_{k}^{>}\left(\eta, \eta^{\prime}\right)$ for $\eta>\eta^{\prime}, G^{>}=G^{<*}$ and other equivalent relations, this can be simplified to give

$$
\begin{aligned}
\left\langle\varphi_{\mathbf{k}_{1}} \varphi_{\mathbf{k}_{2}} \varphi_{\mathbf{k}_{3}} \varphi_{\mathbf{k}_{4}}\right\rangle_{*}^{\mathrm{GE}} & =(2 \pi)^{3} \delta^{3}\left(\sum_{a} \mathbf{k}_{a}\right) \sum_{s} \epsilon_{i j}^{s}\left(\mathbf{k}_{12}\right) k_{1}^{i} k_{2}^{j} \epsilon_{l m}^{s}\left(\mathbf{k}_{34}\right) k_{3}^{l} k_{4}^{m} \int_{-\infty}^{\eta_{*}} \frac{d \eta}{\eta^{2}} \int_{-\infty}^{\eta} \frac{d \eta^{\prime}}{\eta^{\prime 2}} \\
& \times \operatorname{Im}\left[G_{k_{1}}^{>}\left(\eta_{*}, \eta\right) G_{k_{2}}^{>}\left(\eta_{*}, \eta\right)\right] \cdot \operatorname{Im}\left[G_{k_{3}}^{>}\left(\eta_{*}, \eta^{\prime}\right) G_{k_{4}}^{>}\left(\eta_{*}, \eta^{\prime}\right) F_{k_{12}}^{s>}\left(\eta, \eta^{\prime}\right)\right] \\
& +23 \text { permutations } .
\end{aligned}
$$

To proceed, one makes use of Eqs. (2.15) and (2.19) for the propagators $G_{k}^{>}\left(\eta_{*}, \eta^{\prime}\right)$ and $F_{k}^{s>}\left(\eta, \eta^{\prime}\right)$ in this equation, together with the free mode functions for the scalar field and tensor fluctuations, Eqs. (2.5) and (2.6). In total, for the four-point function we obtain, ${ }^{+}$

$$
\begin{aligned}
\left\langle\varphi_{\mathbf{k}_{1}} \varphi_{\mathbf{k}_{2}} \varphi_{\mathbf{k}_{3}} \varphi_{\mathbf{k}_{4}}\right\rangle_{*}^{\mathrm{GE}} & =(2 \pi)^{3} \delta\left(\sum_{a} \mathbf{k}_{a}\right) \frac{H_{*}^{6}}{\prod_{a}\left(2 k_{a}^{3}\right) k_{12}^{3}} \cdot \sum_{s} \epsilon_{i j}^{s}\left(\mathbf{k}_{12}\right) \epsilon_{l m}^{s}\left(\mathbf{k}_{34}\right) k_{1}^{i} k_{2}^{j} k_{3}^{l} k_{4}^{m} \cdot \mathcal{I}_{1234} \\
+ & 23 \text { permutations }
\end{aligned}
$$

where $\mathcal{I}_{1234}$ is defined as

$$
\begin{aligned}
\mathcal{I}_{1234} \equiv \int_{-\infty}^{\eta_{*}} \frac{d \eta}{\eta^{2}} \int_{-\infty}^{\eta} \frac{d \eta^{\prime}}{\eta^{\prime 2}} \operatorname{Im}\left[\left(1+i k_{1} \eta_{*}\right)\left(1+i k_{2} \eta_{*}\right)\left(1-i k_{1} \eta\right)\left(1-i k_{2} \eta\right) e^{i\left(\eta-\eta_{*}\right)\left(k_{1}+k_{2}\right)}\right] \\
\times \operatorname{Im}\left[\left(1+i k_{3} \eta_{*}\right)\left(1+i k_{4} \eta_{*}\right)\left(1-i k_{3} \eta^{\prime}\right)\left(1-i k_{4} \eta^{\prime}\right)\right. \\
\left.\quad \times\left(1+i k_{12} \eta\right)\left(1-i k_{12} \eta^{\prime}\right) e^{i\left(\eta^{\prime}-\eta_{*}\right)\left(k_{3}+k_{4}\right)} e^{-i\left(\eta-\eta^{\prime}\right) k_{12}}\right]
\end{aligned}
$$

and $k_{t}$ is the sum of the magnitudes of the momenta, $k_{t} \equiv \sum_{a} k_{a}$.

Let us work out the permutations in more detail. Since $\epsilon_{i j}^{s}$ is a symmetric tensor and Eq. (2.22) is invariant under the exchanges $1 \leftrightarrow 2$ and $3 \leftrightarrow 4$, one can rewrite this equation as

$\left\langle\varphi_{\mathbf{k}_{1}} \varphi_{\mathbf{k}_{2}} \varphi_{\mathbf{k}_{3}} \varphi_{\mathbf{k}_{4}}\right\rangle_{*}^{\mathrm{GE}}=(2 \pi)^{3} \delta\left(\sum_{a} \mathbf{k}_{a}\right) \frac{4 H_{*}^{6}}{\prod_{a}\left(2 k_{a}^{3}\right)} \cdot \sum_{s} \sum_{\substack{a<b \\ c<d}} \frac{1}{k_{a b}^{3}} \epsilon_{i j}^{s}\left(\mathbf{k}_{a b}\right) \epsilon_{l m}^{s}\left(\mathbf{k}_{c d}\right) k_{a}^{i} k_{b}^{j} k_{c}^{l} k_{d}^{m} \cdot \mathcal{I}_{a b c d}$,

+ An equivalent - and computationally equivalent - interaction picture formalism was derived by Weinberg [43]. With this formalism the trispectrum can be computed straightforwardly using Eq. (1) of this Ref., expanded to second order in the interactions. 
where the sum is only over different indices $a, b, c, d$, and we have collected an overall symmetry factor of 4 which takes into account the exchanges $a \leftrightarrow b$ and $c \leftrightarrow d$. Rewriting this summation somewhat more explicitly, one arrives at the formula

$$
\begin{aligned}
\left\langle\varphi_{\mathbf{k}_{1}} \varphi_{\mathbf{k}_{2}} \varphi_{\mathbf{k}_{3}} \varphi_{\mathbf{k}_{4}}\right\rangle_{*}^{\mathrm{GE}}= & (2 \pi)^{3} \delta\left(\sum_{a} \mathbf{k}_{a}\right) \frac{4 H_{*}^{6}}{\prod_{a}\left(2 k_{a}^{3}\right)} \\
\times \sum_{s} & {\left[\frac{1}{k_{12}^{3}} \epsilon_{i j}^{s}\left(\mathbf{k}_{12}\right) \epsilon_{l m}^{s}\left(\mathbf{k}_{34}\right) k_{1}^{i} k_{2}^{j} k_{3}^{l} k_{4}^{m} \cdot\left(\mathcal{I}_{1234}+\mathcal{I}_{3412}\right)\right.} \\
& +\frac{1}{k_{13}^{3}} \epsilon_{i j}^{s}\left(\mathbf{k}_{13}\right) \epsilon_{l m}^{s}\left(\mathbf{k}_{24}\right) k_{1}^{i} k_{3}^{j} k_{2}^{l} k_{4}^{m} \cdot\left(\mathcal{I}_{1324}+\mathcal{I}_{2413}\right) \\
& \left.+\frac{1}{k_{14}^{3}} \epsilon_{i j}^{s}\left(\mathbf{k}_{14}\right) \epsilon_{l m}^{s}\left(\mathbf{k}_{23}\right) k_{1}^{i} k_{4}^{j} k_{2}^{l} k_{3}^{m} \cdot\left(\mathcal{I}_{1423}+\mathcal{I}_{2314}\right)\right] .
\end{aligned}
$$

The calculation of $\mathcal{I}_{1234}$ is extremely lengthy but straightforward, and is given explicitly in Appendix A. There are divergent terms in the limit $\eta_{*} \rightarrow 0$ together with a logarithmic dependence on the momenta, but these peculiarities cancel between $\mathcal{I}_{1234}$ and $\mathcal{I}_{3412}$ to give an answer which is finite at late times and depends rationally on the $k_{a}$

$$
\begin{aligned}
\mathcal{I}_{1234}+ & \mathcal{I}_{3412}=\frac{k_{1}+k_{2}}{a_{34}^{2}}\left[\frac{1}{2}\left(a_{34}+k_{12}\right)\left(a_{34}^{2}-2 b_{34}\right)+k_{12}^{2}\left(k_{3}+k_{4}\right)\right]+(1,2 \leftrightarrow 3,4) \\
+ & \frac{k_{1} k_{2}}{k_{t}}\left[\frac{b_{34}}{a_{34}}-k_{12}+\frac{k_{12}}{a_{12}}\left(k_{3} k_{4}-k_{12} \frac{b_{34}}{a_{34}}\right)\left(\frac{1}{k_{t}}+\frac{1}{a_{12}}\right)\right]+(1,2 \leftrightarrow 3,4) \\
& -\frac{k_{12}}{a_{12} a_{34} k_{t}}\left[b_{12} b_{34}+2 k_{12}^{2}\left(\prod_{a} k_{a}\right)\left(\frac{1}{k_{t}^{2}}+\frac{1}{a_{12} a_{34}}+\frac{k_{12}}{k_{t} a_{12} a_{34}}\right)\right],
\end{aligned}
$$

where we have used $k_{12}=k_{34}$ and we have defined

$$
a_{a b} \equiv k_{a}+k_{b}+k_{a b}, \quad b_{a b} \equiv\left(k_{a}+k_{b}\right) k_{a b}+k_{a} k_{b} .
$$

To simplify Eq. (2.25) further, let us work out the polarization factors. To start, we will express $\sum_{s} \epsilon_{i j}^{s}\left(\mathbf{k}_{12}\right) \epsilon_{l m}^{s}\left(\mathbf{k}_{34}\right) k_{1}^{i} k_{2}^{j} k_{3}^{l} k_{4}^{m}$ in terms of the relative angles between the $\mathbf{k}_{a}$ and $\mathbf{k}_{12}$. The polarization tensors $\epsilon_{i j}^{s}$ can be rewritten as

$$
\epsilon_{i j}^{+}=\mathbf{e}_{i} \mathbf{e}_{j}-\overline{\mathbf{e}}_{i} \overline{\mathbf{e}}_{j}, \quad \epsilon_{i j}^{\times}=\mathbf{e}_{i} \overline{\mathbf{e}}_{j}+\overline{\mathbf{e}}_{i} \mathbf{e}_{j},
$$

where $\mathbf{e}$ and $\overline{\mathbf{e}}$ are orthogonal unit vectors perpendicular to $\mathbf{k}_{12}$. It is convenient to write the $\mathbf{k}_{a}$ in a spherical coordinate system having $\left\{\mathbf{e}, \overline{\mathbf{e}}, \hat{\mathbf{k}}_{12}\right\}$ as basis (we have denoted $\hat{\mathbf{k}} \equiv \mathbf{k} / k)$. In this coordinate system one obtains

$$
\mathbf{k}_{a}=k_{a}\left(\sin \theta_{a} \cos \phi_{a}, \sin \theta_{a} \sin \phi_{a}, \cos \theta_{a}\right) \text {, }
$$

where $\cos \theta_{a} \equiv \hat{\mathbf{k}}_{a} \cdot \hat{\mathbf{k}}_{12}$ and $\cos \phi_{a} \equiv \hat{\mathbf{k}}_{a} \cdot \mathbf{e}$. Thus

$$
\begin{aligned}
& \epsilon_{i j}^{+} k_{1}^{i} k_{2}^{j}=k_{1} k_{2} \sin \theta_{1} \sin \theta_{2} \cos \left(\phi_{1}+\phi_{2}\right), \\
& \epsilon_{i j}^{\times} k_{1}^{i} k_{2}^{j}=k_{1} k_{2} \sin \theta_{1} \sin \theta_{2} \sin \left(\phi_{1}+\phi_{2}\right),
\end{aligned}
$$

with an analogous relation holding for $\epsilon_{i j}^{+} k_{3}^{i} k_{4}^{j}$ and $\epsilon_{i j}^{\times} k_{3}^{i} k_{4}^{j}$. Since the projections of $\mathbf{k}_{1}$ and $\mathbf{k}_{2}\left(\mathbf{k}_{3}\right.$ and $\left.\mathbf{k}_{4}\right)$ on the plane orthogonal to $\mathbf{k}_{12}$ have the same amplitude but 
opposite directions, then $k_{2} \sin \theta_{2}=k_{1} \sin \theta_{1}$ and $\phi_{2}=\phi_{1}+\pi\left(k_{4} \sin \theta_{4}=k_{3} \sin \theta_{3}\right.$ and $\left.\phi_{4}=\phi_{3}+\pi\right)$. Using these relations, one finally finds

$$
\sum_{s} \epsilon_{i j}^{s}\left(\mathbf{k}_{12}\right) \epsilon_{l m}^{s}\left(\mathbf{k}_{34}\right) k_{1}^{i} k_{2}^{j} k_{3}^{l} k_{4}^{m}=k_{1}^{2} k_{3}^{2} \sin ^{2} \theta_{1} \sin ^{2} \theta_{3} \cos 2 \chi_{12,34},
$$

where $\chi_{12,34} \equiv \phi_{1}-\phi_{3}$ is the angle between the projections of $\mathbf{k}_{1}$ and $\mathbf{k}_{3}$ on the plane orthogonal to $\mathbf{k}_{12}$. This is also the angle between the two planes formed by $\left\{\mathbf{k}_{1}, \mathbf{k}_{2}\right\}$ and $\left\{\mathbf{k}_{3}, \mathbf{k}_{4}\right\}$. This expression can easily be generalized to the polarization factors in the last two lines of Eq. (2.25).

We now wish to convert our prediction for the scalar four-point correlation function due to graviton exchange into a prediction for the correlation function of the curvature perturbation, $\zeta$. In order to do so, it is sufficient to use the linear relation between $\zeta$ and the scalar field perturbation $\varphi$ at Hubble crossing, i.e., $\zeta=\varphi_{*} / \sqrt{2 \epsilon}$. As shown in Refs. [28, 44, 21], higher order terms in the relation between $\zeta$ and $\varphi_{*}$ generate contributions to the trispectrum that are suppressed by slow-roll parameters with respect to the contribution from graviton exchange. Thus, using Eq. (2.32) and analogous expressions for the other polarization factors, we can rewrite Eq. (2.25) to give

$$
\begin{aligned}
\left\langle\zeta_{\mathbf{k}_{1}} \zeta_{\mathbf{k}_{2}} \zeta_{\mathbf{k}_{3}} \zeta_{\mathbf{k}_{4}}\right\rangle^{\mathrm{GE}} & =(2 \pi)^{3} \delta\left(\sum_{a} \mathbf{k}_{a}\right) \frac{H_{*}^{6}}{\epsilon^{2} \prod_{a}\left(2 k_{a}^{3}\right)} \\
\times & {\left[\frac{k_{1}^{2} k_{3}^{2}}{k_{12}^{3}}\left[1-\left(\hat{\mathbf{k}}_{1} \cdot \hat{\mathbf{k}}_{12}\right)^{2}\right]\left[1-\left(\hat{\mathbf{k}}_{3} \cdot \hat{\mathbf{k}}_{12}\right)^{2}\right] \cos 2 \chi_{12,34} \cdot\left(\mathcal{I}_{1234}+\mathcal{I}_{3412}\right)\right.} \\
& +\frac{k_{1}^{2} k_{2}^{2}}{k_{13}^{3}}\left[1-\left(\hat{\mathbf{k}}_{1} \cdot \hat{\mathbf{k}}_{13}\right)^{2}\right]\left[1-\left(\hat{\mathbf{k}}_{2} \cdot \hat{\mathbf{k}}_{13}\right)^{2}\right] \cos 2 \chi_{13,24} \cdot\left(\mathcal{I}_{1324}+\mathcal{I}_{2413}\right) \\
& \left.+\frac{k_{1}^{2} k_{2}^{2}}{k_{14}^{3}}\left[1-\left(\hat{\mathbf{k}}_{1} \cdot \hat{\mathbf{k}}_{14}\right)^{2}\right]\left[1-\left(\hat{\mathbf{k}}_{2} \cdot \hat{\mathbf{k}}_{14}\right)^{2}\right] \cos 2 \chi_{14,23} \cdot\left(\mathcal{I}_{1423}+\mathcal{I}_{2314}\right)\right] .
\end{aligned}
$$

\section{The counter-collinear limit}

The calculation of the four-point correlation induced by the graviton exchange process is rather complicated, and the final expression given in Eq. (2.33) - combined with Eq. (2.26) - is not simple to interpret. One would therefore like an independent check that the result is correct. It is possible to achieve this in the counter-collinear limit, where $\mathbf{k}_{a b}=\mathbf{k}_{a}+\mathbf{k}_{b} \rightarrow 0$ and we can attach a simple physical meaning to the exchange process: in this limit, the four-point function simply expresses the correlation between a pair of two-point functions, for instance $\left\langle\zeta_{\mathbf{k}_{1}} \zeta_{\mathbf{k}_{2}}\right\rangle$ and $\left\langle\zeta_{\mathbf{k}_{3}} \zeta_{\mathbf{k}_{4}}\right\rangle$ which is induced by a low frequency gravitational wave of momentum $k_{12} \ll k_{1} \approx k_{2}, k_{3} \approx k_{4}$. This gravitational wave crossed the Hubble radius much earlier than any of the $k_{a}$ modes and can be considered as a fixed classical background into which they exit [11]. As discussed in $\S 1$, in this limit the momentum quadrilateral constitutes a planar parallelogram (or a folded-kite). 
Exactly the same reasoning applies where the exchanged particle is a scalar mode, and allows us to estimate the size of the contribution from such a process in the countercollinear limit. Following Ref. [26] (see also Ref. [45]), we write the background mode as $\zeta_{B}\left(\mathbf{k}_{0}\right)$. The effect of this mode is to re-scale the spatial coordinates so that the two-point function $\left\langle\zeta_{\mathbf{k}_{1}} \zeta_{\mathbf{k}_{2}}\right\rangle$ receives a correction proportional to its tilt,

$\left\langle\zeta_{\mathbf{k}_{1}} \zeta_{\mathbf{k}_{2}}\right\rangle_{\zeta_{B}}=\left\langle\zeta_{\mathbf{k}_{1}} \zeta_{\mathbf{k}_{2}}\right\rangle-\left(n_{s}-1\right) \int \frac{d^{3} k_{0}}{(2 \pi)^{3}} \zeta_{B}\left(\mathbf{k}_{0}\right)(2 \pi)^{3} \delta\left(\mathbf{k}_{1}+\mathbf{k}_{2}-\mathbf{k}_{0}\right) P_{\zeta}\left(k_{2}\right)$.

A similar formula gives the transformation of $\left\langle\zeta_{\mathbf{k}_{3}} \zeta_{\mathbf{k}_{4}}\right\rangle$. To obtain the contribution to the four-point function due to the scalar background mode (which we label with the superscript "SE") in the counter-collinear limit, it follows that we need only to average over this pair of two-point functions, which yields

$\left\langle\left\langle\zeta_{\mathbf{k}_{1}} \zeta_{\mathbf{k}_{2}}\right\rangle_{\zeta_{B}}\left\langle\zeta_{\mathbf{k}_{3}} \zeta_{\mathbf{k}_{4}}\right\rangle_{\zeta_{B}}\right\rangle^{\mathrm{SE}}=(2 \pi)^{3} \delta\left(\sum \mathbf{k}_{a}\right) \cdot\left(n_{s}-1\right)^{2} \cdot P_{\zeta}\left(k_{12}\right) P_{\zeta}\left(k_{1}\right) P_{\zeta}\left(k_{3}\right)$.

Thus, in the counter-collinear limit, the correlations induced by scalar exchange are small, being suppressed by two powers of the scalar tilt. This is consistent with the estimate made in the Introduction $(\S 1)$, which found the contribution from scalar exchange to be suppressed by $\mathrm{O}\left(\epsilon^{2}, \eta^{2}, \epsilon \eta\right)$. Note that Eq. (3.2) can be thought of as arising from the product of two back-to-back bispectra in the squeezed limit, and exactly satisfies the classical relationship between $\tau_{\mathrm{NL}}$ and $f_{\mathrm{NL}}$ described in Eq. (1.6). Nevertheless, the full trispectrum does not. Indeed, as we show below, although the contributions from the contact interactions are subdominant in this limit [28], those from gravitational wave exchange remain important and dominate the trispectrum.

Now consider the effect of a long wavelength gravitational wave on $\left\langle\zeta_{\mathbf{k}_{1}} \zeta_{\mathbf{k}_{2}}\right\rangle$. This will turn out to be much larger than the effect due to the scalar mode. In this case the effect of the background gravitational wave, $\gamma_{B}^{i j}\left(\mathbf{k}_{0}\right)$, is to deform the geometry in such a way that a small spatial distance $d x^{2}$ is rescaled to $d x^{2} \rightarrow\left(e^{\gamma}\right)_{i j} d x^{i} d x^{j} \simeq d x^{2}+\gamma_{i j} d x^{i} d x^{j}$. In Fourier space we have

$$
\left\langle\zeta_{\mathbf{k}_{1}} \zeta_{\mathbf{k}_{2}}\right\rangle_{\gamma_{B}}=\left\langle\zeta_{\mathbf{k}_{1}} \zeta_{\mathbf{k}_{2}}\right\rangle+\int \frac{d^{3} k_{0}}{(2 \pi)^{3}} \gamma_{B}^{i j}\left(\mathbf{k}_{0}\right)\left(\frac{\partial}{\partial \gamma_{B}^{i j}}\left\langle\zeta_{\tilde{\mathbf{k}}_{1}} \zeta_{\tilde{\mathbf{k}}_{2}}\right\rangle_{\gamma_{B}}\right)_{\gamma_{B}=0},
$$

where $\tilde{\mathbf{k}}_{a}=\mathbf{k}_{a}-\mathbf{k}_{0} / 2$. The effect of the background tensor mode is to deform the momenta such that $k^{2} \rightarrow k^{2}-\gamma_{i j} k^{i} k^{j}$ [11], so that the equation above becomes

$$
\begin{aligned}
&\left\langle\zeta_{\mathbf{k}_{1}} \zeta_{\mathbf{k}_{2}}\right\rangle_{\gamma_{B}}=\left\langle\zeta_{\mathbf{k}_{1}} \zeta_{\mathbf{k}_{2}}\right\rangle-\int \frac{d^{3} k_{0}}{(2 \pi)^{3}} \gamma_{B}^{i j}\left(\mathbf{k}_{0}\right) k_{i}^{1} k_{j}^{1}\left(\frac{\partial}{\partial\left(k^{1}\right)^{2}}\left\langle\zeta_{\tilde{\mathbf{k}}_{1}} \zeta_{\tilde{\mathbf{k}}_{2}}\right\rangle_{\gamma_{B}}\right)_{\gamma_{B}=0} \\
&=\left\langle\zeta_{\mathbf{k}_{1}} \zeta_{\mathbf{k}_{2}}\right\rangle-\int \frac{d^{3} k_{0}}{(2 \pi)^{3}}(2 \pi)^{3} \delta\left(\mathbf{k}_{1}+\mathbf{k}_{2}-\mathbf{k}_{0}\right) \gamma_{B}^{i j}\left(\mathbf{k}_{0}\right) \frac{k_{i}^{1} k_{j}^{1}}{k_{1}^{2}} \frac{3}{2} P_{\zeta}\left(k_{1}\right) .
\end{aligned}
$$

In this case, the effect of deforming the geometry is not suppressed by the scalar tilt, and therefore dominates compared with the effect of a background scalar mode. Nevertheless, Eq. (3.4) is proportional to the amplitude of gravitational waves, which is of order $\sim r^{-1 / 2}$ when compared with the scalar amplitude. For this reason, one finds that the trispectrum in this limit is proportional to the ratio of the tensor to scalar power spectra. 
Indeed, taking the average of $\left\langle\zeta_{\mathbf{k}_{1}} \zeta_{\mathbf{k}_{2}}\right\rangle_{\gamma_{B}}$ and $\left\langle\zeta_{\mathbf{k}_{3}} \zeta_{\mathbf{k}_{4}}\right\rangle_{\gamma_{B}}$ we find the relation

$$
\begin{aligned}
& \left\langle\left\langle\zeta_{\mathbf{k}_{1}} \zeta_{\mathbf{k}_{2}}\right\rangle_{\gamma_{B}}\left\langle\zeta_{\mathbf{k}_{3}} \zeta_{\mathbf{k}_{4}}\right\rangle_{\gamma_{B}}\right\rangle^{\mathrm{GE}}=(2 \pi)^{3} \delta\left(\sum_{a} \mathbf{k}_{a}\right) \\
& \quad \times \sum_{s} \epsilon_{i j}^{s}\left(\mathbf{k}_{12}\right) \frac{k_{1}^{i} k_{1}^{j}}{k_{1}^{2}} \epsilon_{l m}^{s}\left(\mathbf{k}_{34}\right) \frac{k_{3}^{l} k_{3}^{m}}{k_{3}^{2}} \cdot 4 \epsilon \cdot \frac{9}{4} \cdot P_{\zeta}\left(k_{12}\right) P_{\zeta}\left(k_{1}\right) P_{\zeta}\left(k_{3}\right) .
\end{aligned}
$$

We can use Eq. (2.32) to rewrite the polarization factor in this equation. In the limit $k_{12} \rightarrow 0, \theta_{1}, \theta_{3} \rightarrow \pi$ and Eq. (2.32) reads

$$
\sum_{s} \epsilon_{i j}^{s}\left(\mathbf{k}_{12}\right) k_{1}^{i} k_{1}^{j} \epsilon_{l m}^{s}\left(\mathbf{k}_{34}\right) k_{3}^{l} k_{3}^{m}=k_{1}^{2} k_{3}^{2} \cos 2 \chi_{12,34}
$$

Thus, Eq. (3.5) yields a semiclassical relation in the counter-collinear limit $\left(k_{12} \ll k_{1} \approx\right.$ $\left.k_{2}, k_{3} \approx k_{4}\right)$

$$
\left\langle\zeta_{\mathbf{k}_{1}} \zeta_{\mathbf{k}_{2}} \zeta_{\mathbf{k}_{3}} \zeta_{\mathbf{k}_{4}}\right\rangle^{\mathrm{GE}}=(2 \pi)^{3} \delta\left(\sum_{a} \mathbf{k}_{a}\right) \cdot\left(\frac{9}{16} r \cos 2 \chi_{12,34}\right) \cdot P_{\zeta}\left(k_{1}\right) P_{\zeta}\left(k_{3}\right) P_{\zeta}\left(k_{12}\right)
$$

where we have used $r=16 \epsilon$ and in this degenerate limit $\chi_{12,34}$ becomes the angle between $\mathbf{k}_{1}$ and $\mathbf{k}_{3}$.

Let us check that Eq. (2.33) satisfies this relation and consistently reproduces Eq. (3.7) in the counter-collinear limit. Indeed, for $k_{12} \ll k_{1} \approx k_{2}, k_{3} \approx k_{4}$ the second line of Eq. (2.33) dominates over the last two and we must only compute the sum of the integrals $\mathcal{I}_{1234}+\mathcal{I}_{3412}$ by using Eq. (2.26) in this limit. For $k_{12}=0, k_{1}=k_{2}$ and $k_{3}=k_{4}$. Using Eq. (2.27), $a_{12}=2 k_{1}, a_{34}=2 k_{3}, b_{12}=k_{1}^{2}$ and $b_{34}=k_{3}^{2}$. Thus, the right hand side of the first line of Eq. (2.26) yields $2 k_{1} k_{3}$, the second line yields $k_{1} k_{3} / 4$ while the third line vanishes. Then, one finds $\mathcal{I}_{1234}+\mathcal{I}_{3412}=\frac{9}{4} k_{1} k_{3}$ and Eq. (2.33) becomes

$$
\left\langle\zeta_{\mathbf{k}_{1}} \zeta_{\mathbf{k}_{2}} \zeta_{\mathbf{k}_{3}} \zeta_{\mathbf{k}_{4}}\right\rangle^{\mathrm{GE}}=(2 \pi)^{3} \delta\left(\sum_{a} \mathbf{k}_{a}\right) \frac{H_{*}^{6}}{\epsilon^{2} 2^{4} k_{1}^{3} k_{3}^{3} k_{12}^{3}} \cdot \frac{9}{4} \cos 2 \chi_{12,34}
$$

Comparing this equation with (3.7) after using the relations $P_{\zeta}(k)=H_{*}^{2} /\left(4 \epsilon k^{3}\right)$ and $r=16 \epsilon$, one sees that the semiclassical relation is satisfied.

Note that, strictly speaking, Eq. (3.7) is not a consistency relation like Maldacena's consistency relation in the squeezed limit. In particular, the full trispectrum need not satisfy this relation. This relation will be satisfied by the full trispectrum only when contact interactions are negligible with respect to the graviton exchange in the countercollinear limit. Accidentally, as we will show below, this is the case for slow-roll inflation, and the full trispectrum satisfies this relation. However, this is not the case, for instance, in models with non-standard kinetic terms.

\section{The total trispectrum}

The connected four-point correlation function of field perturbations at horizon crossing was computed in Ref. [28], assuming that inflation was driven by a collection of light scalar fields with canonically normalized kinetic terms. However, this calculation was restricted only to contact terms, neglecting correlations induced by particle exchange. 
In this section we put together the trispectrum of Ref. [28] for a single field with the exchange contribution computed in $\S 2$. This gives the full trispectrum to leading order in slow-roll. Then we discuss this result.

When specialized to the case of a single field, the contact interaction (labelled with a superscript "CI") gives a contribution to the scalar trispectrum equal to [28]

$$
\left\langle\zeta_{\mathbf{k}_{1}} \zeta_{\mathbf{k}_{2}} \zeta_{\mathbf{k}_{3}} \zeta_{\mathbf{k}_{4}}\right\rangle^{\mathrm{CI}}=(2 \pi)^{3} \delta\left(\sum_{a} \mathbf{k}_{a}\right) \frac{H_{*}^{6}}{4 \epsilon^{2} \prod_{a}\left(2 k_{a}^{3}\right)} \sum_{\text {perms }} \mathcal{M}_{4}\left(\mathbf{k}_{1}, \mathbf{k}_{2}, \mathbf{k}_{3}, \mathbf{k}_{4}\right)
$$

where we have defined a form-factor $\mathcal{M}_{4}$ which encapsulates the detailed momentum dependence. This is given by the expression

$$
\begin{gathered}
\mathcal{M}_{4}\left(\mathbf{k}_{1}, \mathbf{k}_{2}, \mathbf{k}_{3}, \mathbf{k}_{4}\right)=-2 \frac{k_{1}^{2} k_{3}^{2}}{k_{12}^{2} k_{34}^{2}} \frac{W_{24}}{k_{t}}\left(\frac{\mathbf{Z}_{12} \cdot \mathbf{Z}_{34}}{k_{34}^{2}}+2 \mathbf{k}_{2} \cdot \mathbf{Z}_{34}+\frac{3}{4} \sigma_{12} \sigma_{34}\right) \\
-\frac{1}{2} \frac{k_{3}^{2}}{k_{34}^{2}} \sigma_{34}\left(\frac{\mathbf{k}_{1} \cdot \mathbf{k}_{2}}{k_{t}} W_{124}+2 \frac{k_{1}^{2} k_{2}^{2}}{k_{t}^{3}}+6 \frac{k_{1}^{2} k_{2}^{2} k_{4}}{k_{t}^{4}}\right),
\end{gathered}
$$

where the quantities occurring here are defined by

$$
\begin{aligned}
\sigma_{a b} & =\mathbf{k}_{a} \cdot \mathbf{k}_{b}+k_{b}^{2}, \\
\mathbf{Z}_{a b} & =\sigma_{a b} \mathbf{k}_{a}-\sigma_{b a} \mathbf{k}_{b}, \\
W_{a b} & =1+\frac{k_{a}+k_{b}}{k_{t}}+\frac{2 k_{a} k_{b}}{k_{t}^{2}}, \\
W_{a b c} & =1+\frac{k_{a}+k_{b}+k_{c}}{k_{t}}+\frac{2\left(k_{a} k_{b}+k_{b} k_{c}+k_{a} k_{c}\right)}{k_{t}^{2}}+\frac{6 k_{a} k_{b} k_{c}}{k_{t}^{3}} .
\end{aligned}
$$

In order to write Eq. (4.1) from Ref. [28], we have used the linear relation $\zeta=\varphi_{*} / \sqrt{2 \epsilon}$ to express $\zeta$ in terms of the field perturbations at Hubble crossing $\varphi_{*}$. As discussed earlier, nonlinearities in the relation between $\zeta$ and $\varphi_{*}$ lead to corrections to the trispectrum that are suppressed by slow-roll with respect to $(4.1)[28,44,21]$. Thus, at leading order in slow-roll, the total trispectrum is given by the sum of the two contributions, Eqs. (2.33) and (4.1),

$$
\left\langle\zeta_{\mathbf{k}_{1}} \zeta_{\mathbf{k}_{2}} \zeta_{\mathbf{k}_{3}} \zeta_{\mathbf{k}_{4}}\right\rangle=\left\langle\zeta_{\mathbf{k}_{1}} \zeta_{\mathbf{k}_{2}} \zeta_{\mathbf{k}_{3}} \zeta_{\mathbf{k}_{4}}\right\rangle_{*}^{\mathrm{CI}}+\left\langle\zeta_{\mathbf{k}_{1}} \zeta_{\mathbf{k}_{2}} \zeta_{\mathbf{k}_{3}} \zeta_{\mathbf{k}_{4}}\right\rangle_{*}^{\mathrm{GE}}
$$

We have already discussed the squeezed limit, in which one of the momenta becomes much smaller than the others. In this limit, the trispectrum must be described by Maldacena's consistency relation [11, 46, 26, 47, 45]: the scalar mode associated with the small momentum forms a fixed classical background into which the remaining modes exit, in a similar fashion to the calculations discussed in $§ 3$. In Ref. [28] it was noted that the scalar contact term is one order lower in slow-roll than the term yielded by the consistency relation in this limit. These two apparently contradictory statements are nevertheless in agreement, because the leading-order slow-roll piece in the contact term vanishes in the squeezed limit. Although we have not pursued the calculation of the trispectrum to a sufficiently high order in the slow-roll to verify that Maldacena's relation holds in detail for the graviton exchange process, our final result for the trispectrum, Eq. (4.7), is also one order lower in slow-roll than predicted by the consistency relation, but vanishes in the squeezed limit. It is therefore consistent with Maldacena's result. 
In the counter-collinear limit $k_{12} \ll k_{1} \approx k_{2}, k_{3} \approx k_{4}$ the form-factor $\mathcal{M}_{4}$ defined in Eq. (4.2) remains finite [28], so that the total trispectrum in Eq. (4.7) is dominated by the contribution from graviton exchange. One can show that in this limit it is possible to parameterize the total trispectrum (4.7) in terms of the local parameter $\tau_{\mathrm{NL}}^{\text {local }}$. Indeed, neglecting the contribution from contact interactions, the total trispectrum is given by Eq. (3.7). Thus, comparing this equation with Eq. (1.9) gives

$$
\tau_{\mathrm{NL}}^{\text {local }}=\frac{9}{64} r \cos 2 \chi_{12,34}
$$

Thus, in the counter-collinear limit, the trispectrum of single-field slow-roll inflation is of the local type in real space, with a magnitude expressed by Eq. (4.8). This is consistent with our expectations: indeed, as was discussed in the Introduction $(\S 1)$, we expect classical processes operating outside the horizon to synthesize local contributions to the curvature perturbation. The counter-collinear limit of graviton exchange is tantamount to such a process: it corresponds to a gravitational wave which nucleates inside the horizon in the far past of inflation, and subsequently passes beyond the Hubble radius where it becomes approximately classical. In the diagrammatic interpretation, this extremely soft graviton then splits into four scalar quanta which propagate to their own hypersurface of horizon exit. These four quanta are correlated because they all originate from the same parent graviton, or gravitational wave.

\section{Discussion and conclusion}

We have calculated a new contribution to the inflationary trispectrum, arising from a process which can be visualized as graviton exchange. This contribution is of the same order of magnitude as the one previously calculated by considering only scalar and vector perturbations and gives rise to an effect of magnitude $\tau_{\mathrm{NL}} \sim \epsilon$. Although one might have expected $\tau_{\mathrm{NL}} \sim \mathrm{O}\left(\epsilon^{2}, \eta^{2}, \epsilon \eta\right)$ on the basis of Maldacena's consistency relation, we can conclude that the estimate $\tau_{\mathrm{NL}} \sim \epsilon$ made in Ref. [28] is essentially correct, although modified in detail by numerical factors coming from the exchange process which was neglected in that work. Note that although we have framed our calculations in the context of single-field inflation, our results for the curvature perturbation also hold for models with multiple fields and a flat field space metric.

We would like to emphasize that the relation $\tau_{\mathrm{NL}} \sim f_{\mathrm{NL}}^{2}$ [see Eq. (1.7)], which

one might anticipate based on comparison of the local formulae (1.3) and (1.6), need not apply. Indeed, it does not apply in single-field slow-roll inflation models. As explained in the Introduction, one can only trust such classical relationships if the non-Gaussianity is generated by gravitational evolution subsequent to horizon crossing. In single-field inflation, non-Gaussianities are a quantum interference effect and are produced at Hubble crossing, after which the curvature perturbation $\zeta$ remains constant.

In Ref. [28], it was supposed that the effect from quantum processes around horizon crossing would be maximized on a configuration close to the equilateral case, where all 
$k_{a}$ take the same magnitude $k$. In the exactly equilateral limit $\left|\tau_{\mathrm{NL}}\right|$ can be computed analytically, giving a maximum effect which corresponds to

$$
\left.\tau_{\mathrm{NL}}^{\mathrm{CI}} \leqslant \frac{23}{576 \sqrt{3}} r \approx 0.0231 r \approx \frac{r}{44} \quad \text { (equilateral and } g_{\mathrm{NL}}=0\right),
$$

where to compute $\tau_{\mathrm{NL}}$ from the trispectrum we have set $g_{\mathrm{NL}}=0$. The tensor to scalar ratio $r$ is currently subject to the observational constraint $r<0.22$ at 95\% CL from a combination of the WMAP five-year, baryon oscillation and supernova data [48]. (This bound was approximated as $\tau_{\mathrm{NL}} \lesssim r / 50$ in Ref. [28].) By searching through the available parameter space, we have subsequently located a marginally off-equilateral configuration which gives a non-linearity of greater magnitude, but opposite sign,

$$
\tau_{\mathrm{NL}}^{\mathrm{CI}}=0.0298 r \approx \frac{r}{34} \quad\left(\text { side ratios } 1: 1: 1: 2.96 \text { and } g_{\mathrm{NL}}=0\right) .
$$

How large a correlation can be induced by the effect of graviton exchange? It is difficult to pursue analytic estimates for the graviton exchange contribution. Proceeding numerically, however, it is possible to find a marginally squeezed configuration which gives an effect corresponding to

$$
\tau_{\mathrm{NL}}^{\mathrm{GE}}=-1.217 r \quad\left(\text { side ratios } 1: 10: 10: 13.44 \text { and } g_{\mathrm{NL}}=0\right) \text {. }
$$

From Eqs. (5.2) and (5.3), it is clear that the total trispectrum will be dominated by the contribution from graviton exchange. Taking both effects together, we have located a configuration which gives a combined $\tau_{\mathrm{NL}}$ equivalent to

$$
\left.\tau_{\mathrm{NL}}=-1.218 r \quad \text { (side ratios } 1: 5.6: 10: 10 \text { and } g_{\mathrm{NL}}=0\right) .
$$

We would like to emphasize, however, that our numerical search has not been exhaustive, and it is possible that other configurations exist which give even larger $\left|\tau_{\mathrm{NL}}\right|$. These estimates hold for multiple-field inflation provided that the hierarchy among the sides of the momentum quadrilateral is not too large [28]. For instance, the ratio of sides given in Eq. (5.3) corresponds to an interval of $\sim 2.6$ e-folds between horizon exit of the modes corresponding to largest and smallest wavelengths. For such a small interval, we can expect that the approximation of almost simultaneous horizon exit is reasonably satisfied. On the other hand, in the limit where a large hierarchy exists among the ratio of sides in the quadrilateral, our analytical expression becomes untrustworthy.

We can therefore conclude that the trispectrum at horizon crossing is generally dominated by the contribution from graviton exchange, and that at maximum the total trispectrum is roughly of order $\left|\tau_{\mathrm{NL}}\right| \sim r$.

\section{Acknowledgments}

It is a pleasure to acknowledge helpful discussions with Frederico Arroja, Paolo Creminelli, Kazuya Koyama and Marcello Musso. We would like to thank Peter Adshead, Richard Easther and Eugene Lim for very interesting correspondence, and for spotting several typos in earlier versions of this paper. 
FV would like to thank the Department of Physics and Astronomy of the University of Aarhus, where this work was initiated, for their kind hospitality. Furthermore, we would like to thank the Department of Applied Mathematics and Theoretical Physics at Cambridge for their hospitality while this work was being carried out - during the workshop Non-Gaussianity from fundamental physics held in September 2008 - and the EU Marie Curie Research \& Training network "UniverseNet" (MRTN-CT-2006-035863) for support. MSS would also like to thank the Institute of Theoretical Astrophysics in Oslo for their hospitality during Fall 2008. DS is supported by STFC.

\section{Appendix A. Computing the time integral}

In this Appendix we compute the sum of the time integrals $\mathcal{I}_{1234}+\mathcal{I}_{3412}$. Let us start by computing $\mathcal{I}_{1234}$, which reads

$$
\begin{aligned}
\mathcal{I}_{1234} \equiv \int_{-\infty}^{\eta_{*}} \frac{d \eta}{\eta^{2}} \int_{-\infty}^{\eta} \frac{d \eta^{\prime}}{\eta^{\prime 2}} \operatorname{Im}\left[\left(1+i k_{1} \eta_{*}\right)\left(1+i k_{2} \eta_{*}\right)\left(1-i k_{1} \eta\right)\left(1-i k_{2} \eta\right) e^{i\left(\eta-\eta_{*}\right)\left(k_{1}+k_{2}\right)}\right] \\
\times \operatorname{Im}\left[\left(1+i k_{3} \eta_{*}\right)\left(1+i k_{4} \eta_{*}\right)\left(1-i k_{3} \eta^{\prime}\right)\left(1-i k_{4} \eta^{\prime}\right)\right. \\
\left.\quad \times\left(1+i k_{12} \eta\right)\left(1-i k_{12} \eta^{\prime}\right) e^{i\left(\eta^{\prime}-\eta_{*}\right)\left(k_{3}+k_{4}\right)} e^{-i\left(\eta-\eta^{\prime}\right) k_{12}}\right]
\end{aligned}
$$

It is convenient to divide this into the sum of 4 integrals. Using the rule $\operatorname{Im}(A B)=$ $-\mathrm{i}\left(A B^{*}-A^{*} B\right) / 2$ we obtain

$$
\mathcal{I}_{1234}=-\frac{1}{4}\left(I_{A}-I_{B}-I_{C}+I_{D}\right)
$$

where

$$
\begin{aligned}
I_{A} & \equiv \int_{-\infty}^{\eta_{*}} \frac{d \eta}{\eta^{2}} X Y \int_{-\infty}^{\eta} \frac{d \eta^{\prime}}{\eta^{\prime 2}} Z, \\
I_{B} & \equiv \int_{-\infty}^{\eta_{*}} \frac{d \eta}{\eta^{2}} X^{*} Y \int_{-\infty}^{\eta} \frac{d \eta^{\prime}}{\eta^{\prime 2}} Z, \\
I_{C} & \equiv \int_{-\infty}^{\eta_{*}} \frac{d \eta}{\eta^{2}} X Y^{*} \int_{-\infty}^{\eta} \frac{d \eta^{\prime}}{\eta^{\prime 2}} Z^{*}, \\
I_{D} & \equiv \int_{-\infty}^{\eta_{*}} \frac{d \eta}{\eta^{2}} X^{*} Y^{*} \int_{-\infty}^{\eta} \frac{d \eta^{\prime}}{\eta^{\prime 2}} Z^{*},
\end{aligned}
$$

and

$$
\begin{aligned}
& X\left(\eta, \eta_{*}\right) \equiv\left(1+i k_{1} \eta_{*}\right)\left(1+i k_{2} \eta_{*}\right)\left(1-i k_{1} \eta\right)\left(1-i k_{2} \eta\right) e^{i\left(\eta-\eta_{*}\right)\left(k_{1}+k_{2}\right)} \\
& Y\left(\eta, \eta_{*}\right) \equiv\left(1+i k_{12} \eta\right) e^{-i \eta k_{12}} \\
& Z\left(\eta^{\prime}, \eta_{*}\right) \equiv\left(1+i k_{3} \eta_{*}\right)\left(1+i k_{4} \eta_{*}\right)\left(1-i k_{3} \eta^{\prime}\right)\left(1-i k_{4} \eta^{\prime}\right)\left(1-i k_{12} \eta^{\prime}\right) e^{i\left(\eta^{\prime}-\eta_{*}\right)\left(k_{3}+k_{4}\right)+i \eta^{\prime} k_{12}}
\end{aligned}
$$

Each of these have to be solved separately.

Let us first consider $I_{A}$. Expanding the $\eta_{*}$-dependent part near $\eta_{*} \rightarrow 0$, this can be written in the form

$$
I_{A}=\left(1+i \delta \eta_{*}-\frac{\delta^{2}}{2} \eta_{*}^{2}+\frac{1}{2} \sum_{a} k_{a}^{2} \eta_{*}^{2}+\ldots\right) e^{-i \eta_{*} \delta}
$$


Inflationary trispectrum from graviton exchange

$$
\begin{aligned}
& \times \int_{-\infty}^{\eta_{*}} \frac{\mathrm{d} \eta}{\eta^{2}}\left(1-\mathrm{i} \alpha_{1} \eta+\alpha_{2} \eta^{2}-\mathrm{i} \alpha_{3} \eta^{3}\right) \mathrm{e}^{\mathrm{i} \eta \alpha_{1}} \\
& \times \int_{-\infty}^{\eta} \frac{\mathrm{d} \eta^{\prime}}{\eta^{\prime 2}}\left(1-\mathrm{i} \beta_{1} \eta^{\prime}+\beta_{2} \eta^{\prime 2}-\mathrm{i} \beta_{3} \eta^{\prime 3}\right) \mathrm{e}^{\mathrm{i} \eta^{\prime} \beta_{1}}
\end{aligned}
$$

where

$$
\begin{aligned}
& \alpha_{1} \equiv k_{1}+k_{2}-k_{12}, \\
& \alpha_{2} \equiv\left(k_{1}+k_{2}\right) k_{12}-k_{1} k_{2}, \\
& \alpha_{3} \equiv k_{1} k_{2} k_{12}, \\
& \beta_{1} \equiv k_{3}+k_{4}+k_{12}, \\
& \beta_{2} \equiv-\left(k_{3}+k_{4}\right) k_{12}-k_{3} k_{4}, \\
& \beta_{3} \equiv-k_{3} k_{4} k_{12}, \\
& \delta \equiv \alpha_{1}+\beta_{1} .
\end{aligned}
$$

The inner integral in the last line of Eq. (A.10) can be easily solved and its log divergent part cancels out. Plugging its solution into Eq. (A.10) yields

$$
\begin{aligned}
I_{A}=\left(1+i \delta \eta_{*}\right. & \left.-\frac{\delta^{2}}{2} \eta_{*}^{2}+\frac{1}{2} \sum_{a} k_{a}^{2} \eta_{*}^{2}+\ldots\right) e^{-i \eta_{*} \delta} \\
& \times \int_{-\infty}^{\eta_{*}} \mathrm{~d} \eta\left(-\frac{1}{\eta^{3}}+\frac{\mathrm{i} \gamma_{1}}{\eta^{2}}+\frac{\gamma_{2}}{\eta}+\mathrm{i} \gamma_{3}+\gamma_{4} \eta+\mathrm{i} \gamma_{5} \eta^{2}\right) \mathrm{e}^{\mathrm{i} \eta \delta},
\end{aligned}
$$

where the $\gamma$ 's are defined by

$$
\begin{aligned}
& \gamma_{1} \equiv \alpha_{1}-\frac{\beta_{1} \beta_{2}+\beta_{3}}{\beta_{1}^{2}}, \\
& \gamma_{2} \equiv-\frac{\alpha_{1}}{\beta_{1}^{2}}\left(\beta_{1} \beta_{2}+\beta_{3}\right)-\alpha_{2}-\frac{\beta_{3}}{\beta_{1}} \\
& \gamma_{3} \equiv \alpha_{3}-\frac{\alpha_{2}}{\beta_{1}^{2}}\left(\beta_{1} \beta_{2}+\beta_{3}\right)+\frac{\alpha_{1} \beta_{3}}{\beta_{1}}, \\
& \gamma_{4} \equiv-\frac{\alpha_{3}}{\beta_{1}^{2}}\left(\beta_{1} \beta_{2}+\beta_{3}\right)-\frac{\alpha_{2} \beta_{3}}{\beta_{1}} \\
& \gamma_{5} \equiv \frac{\alpha_{3} \beta_{3}}{\beta_{1}} .
\end{aligned}
$$

Carrying out the remaining $\eta$ integration, the final result is

$$
\begin{aligned}
I_{A}=\delta\left(\gamma_{1}-\frac{3}{4} \delta\right)+\frac{\gamma_{3}}{\delta}+\frac{\gamma_{4}}{\delta^{2}}-2 \frac{\gamma_{5}}{\delta^{3}} & \\
& +\frac{1}{2 \eta_{*}^{2}}+\frac{1}{4} \sum_{a} k_{a}^{2}+\frac{\mathrm{i}}{\eta_{*}}\left(\delta-\gamma_{1}\right)-\left(\gamma_{2}-\delta \gamma_{1}+\frac{\delta^{2}}{2}\right) E_{1}\left(-\mathrm{i} \delta \eta_{*}\right),
\end{aligned}
$$

where $E_{1}$ is the exponential integral or incomplete $\Gamma$ function,

$$
E_{1}(z)=\int_{z}^{\infty} \frac{\mathrm{e}^{-t}}{t} \mathrm{~d} t
$$

This calculation can be repeated for the remaining integrals $I_{B, C, D}$. The final result (A.24) can be generalized to $I_{B}$ by letting $k_{1} \rightarrow-k_{1}$ and $k_{2} \rightarrow-k_{2}$; to $I_{C}$ 
by the replacements $k_{3} \rightarrow-k_{3}, k_{4} \rightarrow-k_{4}$ and $k_{12} \rightarrow-k_{12}$; and to $I_{D}$ by allowing all the $k$ s to change sign. Eq. (A.2) can then be extended to $\mathcal{I}_{3412}$ by exchanging $12 \leftrightarrow 34$.

Note that in the last line of Eq. (A.24) there are terms that diverge for $\eta_{*} \rightarrow 0$. However, we will now show that this line does not contribute to the final combination $\mathcal{I}_{1234}+\mathcal{I}_{3412}$ and can be thus dropped from the discussion. Indeed, the first and second terms in this line are the same for all the four integrals $I_{A, B, C, D}$. Thus they do not contribute to the sum in Eq. (A.2). The third term of this line is odd under exchange of $I_{A} \leftrightarrow I_{D}$ and $I_{B} \leftrightarrow I_{C}$. Thus, this term also does not contribute to the sum (A.2). Furthermore, it is straightforward to show that the last term of this line does not contribute when considering the final sum $\mathcal{I}_{1234}+\mathcal{I}_{3412}$. One might be concerned that Eq. (A.24) contains apparently divergent (and unphysical) terms in the limit $\delta \rightarrow 0$, which occurs when the momentum quadrilateral becomes counter-collinear. Although it is not possible to see cancellation at intermediate stages in the calculation, these terms also cancel in $\mathcal{I}_{1234}+\mathcal{I}_{3412} \cdot *$

We are therefore left with only the first line of Eq. (A.24) which can be computed straightforwardly, by considering the combined contribution from $\mathcal{I}_{1234}$ and $\mathcal{I}_{3412}$. This yields, using $k_{12}=k_{34}$,

$$
\begin{aligned}
\mathcal{I}_{1234}+ & \mathcal{I}_{3412}=\frac{k_{1}+k_{2}}{a_{34}^{2}}\left[\frac{1}{2}\left(a_{34}+k_{12}\right)\left(a_{34}^{2}-2 b_{34}\right)+k_{12}^{2}\left(k_{3}+k_{4}\right)\right]+(1,2 \leftrightarrow 3,4) \\
& +\frac{k_{1} k_{2}}{k_{t}}\left[\frac{b_{34}}{a_{34}}-k_{12}+\frac{k_{12}}{a_{12}}\left(k_{3} k_{4}-k_{12} \frac{b_{34}}{a_{34}}\right)\left(\frac{1}{k_{t}}+\frac{1}{a_{12}}\right)\right]+(1,2 \leftrightarrow 3,4) \\
& -\frac{k_{12}}{a_{12} a_{34} k_{t}}\left[b_{12} b_{34}+2 k_{12}^{2} \prod_{a} k_{a}\left(\frac{1}{k_{t}^{2}}+\frac{1}{a_{12} a_{34}}+\frac{k_{12}}{k_{t} a_{12} a_{34}}\right)\right],
\end{aligned}
$$

where $a_{a b} \equiv k_{a}+k_{b}+k_{a b}$ and $b_{a b} \equiv\left(k_{a}+k_{b}\right) k_{a b}+k_{a} k_{b}$. The first line of Eq. (A.27) comes from summing all the contributions which correspond to the first term in the first line of Eq. (A.24), while the last two lines come from the final three terms.

* Note added after publication. We have chosen to nest the terms arising from $(+,-)$ and $(-,+)$ diagrams, which enter with a minus sign inside the square bracket of Eq. (2.23). The right hand side of Eq. (2.23) could alternatively have been evaluated by factorization, which leads to an expression of the form

$$
\begin{aligned}
\left\langle\varphi_{\mathbf{k}_{1}} \varphi_{\mathbf{k}_{2}} \varphi_{\mathbf{k}_{3}} \varphi_{\mathbf{k}_{4}}\right\rangle_{*}^{\mathrm{GE}} & =-\frac{1}{4}(2 \pi)^{3} \delta^{3}\left(\sum_{a} \mathbf{k}_{a}\right) \sum_{s} \epsilon_{i j}^{s}\left(\mathbf{k}_{12}\right) k_{1}^{i} k_{2}^{j} \epsilon_{l m}^{s}\left(\mathbf{k}_{34}\right) k_{3}^{l} k_{4}^{m} \\
\times & {\left[2 \operatorname{Re}\left(\int_{-\infty}^{\eta_{*}} \frac{d \eta}{\eta^{2}} \int_{-\infty}^{\eta} \frac{d \eta^{\prime}}{\eta^{\prime 2}} G_{k_{1}}^{<}\left(\eta_{*}, \eta\right) G_{k_{2}}^{<}\left(\eta_{*}, \eta\right) G_{k_{3}}^{<}\left(\eta_{*}, \eta^{\prime}\right) G_{k_{4}}^{<}\left(\eta_{*}, \eta^{\prime}\right) F_{k_{12}}^{s<}\left(\eta, \eta^{\prime}\right)\right)\right.} \\
& \left.-\int_{-\infty}^{\eta_{*}} \frac{d \eta}{\eta^{2}} \int_{-\infty}^{\eta_{*}} \frac{d \eta^{\prime}}{\eta^{\prime 2}} G_{k_{1}}^{>}\left(\eta_{*}, \eta\right) G_{k_{2}}^{>}\left(\eta_{*}, \eta\right) G_{k_{3}}^{<}\left(\eta_{*}, \eta^{\prime}\right) G_{k_{4}}^{<}\left(\eta_{*}, \eta^{\prime}\right) F_{k_{12}}^{s<}\left(\eta, \eta^{\prime}\right)\right] \\
& +23 \text { permutations } .
\end{aligned}
$$

Once we explicitly replace the propagators in terms of mode functions, this expression yields no factors of $\delta$ in the denominator. In this case, it is unnecessary to check that the limit $\delta \rightarrow 0$ is regular and the calculation is simplified. We would like to thank Peter Adshead, Richard Easther and Eugene Lim for interesting correspondence on this issue. Note, however, that whichever method one chooses, the final answer is the same. 


\section{References}

[1] C. Hikage et al., Limits on Primordial Non-Gaussianity from Minkowski Functionals of the WMAP Temperature Anisotropies, Mon. Not. Roy. Astron. Soc. 389 (2008) 1439-1446, [0802.3677].

[2] L. Verde, L.-M. Wang, A. Heavens, and M. Kamionkowski, Large-scale structure, the cosmic microwave background, and primordial non-gaussianity, Mon. Not. Roy. Astron. Soc. 313 (2000) L141-L147, [astro-ph/9906301].

[3] E. Komatsu and D. N. Spergel, Acoustic signatures in the primary microwave background bispectrum, Phys. Rev. D63 (2001) 063002, [astro-ph/0005036].

[4] D. Babich, P. Creminelli, and M. Zaldarriaga, The shape of non-Gaussianities, JCAP 0408 (2004) 009, [astro-ph/0405356].

[5] D. H. Lyth and Y. Rodriguez, The inflationary prediction for primordial non-gaussianity, Phys. Rev. Lett. 95 (2005) 121302, [astro-ph/0504045].

[6] N. Bartolo, S. Matarrese, and A. Riotto, Enhancement of non-Gaussianity after inflation, JHEP 04 (2004) 006, [astro-ph/0308088].

[7] N. Bartolo, S. Matarrese, and A. Riotto, CMB Anisotropies at Second Order I, JCAP 0606 (2006) 024, [astro-ph/0604416].

[8] N. Bartolo, S. Matarrese, and A. Riotto, CMB Anisotropies at Second-Order II: Analytical Approach, JCAP 0701 (2007) 019, [astro-ph/0610110].

[9] C. Pitrou, J.-P. Uzan, and F. Bernardeau, Cosmic microwave background bispectrum on small angular scales, 0807.0341.

[10] C. Pitrou, The radiative transfer at second order: a full treatment of the Boltzmann equation with polarization, 0809.3036.

[11] J. M. Maldacena, Non-Gaussian features of primordial fluctuations in single field inflationary models, JHEP 05 (2003) 013, [astro-ph/0210603].

[12] V. Acquaviva, N. Bartolo, S. Matarrese, and A. Riotto, Second-order cosmological perturbations from inflation, Nucl. Phys. B667 (2003) 119-148, [astro-ph/0209156].

[13] D. Babich and M. Zaldarriaga, Primordial Bispectrum Information from CMB Polarization, Phys. Rev. D70 (2004) 083005, [astro-ph/0408455].

[14] W. Hu, Angular trispectrum of the cosmic microwave background, Phys. Rev. D64 (2001) 083005, [astro-ph/0105117].

[15] G. De Troia et al., The trispectrum of the Cosmic Microwave Background on sub-degree angular scales: an analysis of the BOOMERanG data, Mon. Not. Roy. Astron. Soc. 343 (2003) 284, [astro-ph/0301294].

[16] M. Kunz, A. J. Banday, P. G. Castro, P. G. Ferreira, and K. M. Gorski, The Trispectrum of the 4 Year COBE-DMR data, astro-ph/0111250.

[17] A. Cooray, C. Li, and A. Melchiorri, The trispectrum of 21-cm background anisotropies as a probe of primordial non-Gaussianity, Phys. Rev. D77 (2008) 103506, [0801.3463].

[18] G. D'Amico, N. Bartolo, S. Matarrese, and A. Riotto, CMB temperature anisotropies from third order gravitational perturbations, JCAP 0801 (2008) 005, [0707.2894].

[19] T. Okamoto and W. Hu, The Angular Trispectra of CMB Temperature and Polarization, Phys. Rev. D66 (2002) 063008, [astro-ph/0206155].

[20] N. Kogo and E. Komatsu, Angular Trispectrum of CMB Temperature Anisotropy from Primordial Non-Gaussianity with the Full Radiation Transfer Function, Phys. Rev. D73 (2006) 083007, [astro-ph/0602099].

[21] C. T. Byrnes, M. Sasaki, and D. Wands, The primordial trispectrum from inflation, Phys. Rev. D74 (2006) 123519, [astro-ph/0611075].

[22] P. Creminelli, On non-gaussianities in single-field inflation, JCAP 0310 (2003) 003, [astro-ph/0306122].

[23] L. Boubekeur and D. H. Lyth, Detecting a small perturbation through its non-Gaussianity, Phys. 
Rev. D73 (2006) 021301, [astro-ph/0504046].

[24] L. Alabidi and D. H. Lyth, Inflation models and observation, JCAP 0605 (2006) 016, [astro-ph/0510441].

[25] P. Adshead, R. Easther and E. A. Lim, Cosmology With Many Light Scalar Fields: Stochastic Inflation and Loop Corrections, arXiv:0809.4008 [hep-th].

[26] X. Chen, M.-x. Huang, and G. Shiu, The inflationary trispectrum for models with large nonGaussianities, Phys. Rev. D74 (2006) 121301, [hep-th/0610235].

[27] F. Arroja and K. Koyama, Non-gaussianity from the trispectrum in general single field inflation, Phys. Rev. D77 (2008) 083517, [0802.1167].

[28] D. Seery, J. E. Lidsey, and M. S. Sloth, The inflationary trispectrum, JCAP 0701 (2007) 027, [astro-ph/0610210].

[29] P. R. Jarnhus and M. S. Sloth, de Sitter limit of inflation and nonlinear perturbation theory, JCAP 0802 (2008) 013, [0709.2708].

[30] E. Dimastrogiovanni and N. Bartolo, One-loop graviton corrections to the curvature perturbation from inflation, 0807.2790.

[31] M. S. Sloth, On the one loop corrections to inflation and the CMB anisotropies, Nucl. Phys. B748 (2006) 149-169, [astro-ph/0604488].

[32] M. S. Sloth, On the one loop corrections to inflation. II: The consistency relation, Nucl. Phys. B775 (2007) 78-94, [hep-th/0612138].

[33] D. Seery, One-loop corrections to a scalar field during inflation, JCAP 0711 (2007) 025, [0707.3377].

[34] D. Seery, One-loop corrections to the curvature perturbation from inflation, JCAP 0802 (2008) 006, [0707.3378].

[35] Y. Urakawa and K.-i. Maeda, One-loop Corrections to Scalar and Tensor Perturbations during Inflation in Stochastic Gravity, 0801.0126.

[36] R. L. Arnowitt, S. Deser, and C. W. Misner, Canonical variables for general relativity, Phys. Rev. 117 (1960) 1595-1602.

[37] D. H. Lyth, K. A. Malik, and M. Sasaki, A general proof of the conservation of the curvature perturbation, JCAP 0505 (2005) 004, [astro-ph/0411220].

[38] F. Vernizzi, On the conservation of second-order cosmological perturbations in a scalar field dominated Universe, Phys. Rev. D71 (2005) 061301, [astro-ph/0411463].

[39] D. Langlois and F. Vernizzi, Nonlinear perturbations of cosmological scalar fields, JCAP 0702 (2007) 017, [astro-ph/0610064].

[40] J. S. Schwinger, Brownian motion of a quantum oscillator, J. Math. Phys. 2 (1961) 407-432.

[41] L. V. Keldysh, Diagram technique for nonequilibrium processes, Zh. Eksp. Teor. Fiz. 47 (1964) $1515-1527$.

[42] E. Calzetta and B. L. Hu, Closed Time Path Functional Formalism in Curved Space- Time: Application to Cosmological Back Reaction Problems, Phys. Rev. D35 (1987) 495.

[43] S. Weinberg, Quantum contributions to cosmological correlations, Phys. Rev. D72 (2005) 043514, [hep-th/0506236].

[44] D. Seery and J. E. Lidsey, Non-gaussianity from the inflationary trispectrum, JCAP 0701 (2007) 008, [astro-ph/0611034].

[45] C. Cheung, A. L. Fitzpatrick, J. Kaplan, and L. Senatore, On the consistency relation of the 3-point function in single field inflation, JCAP 0802 (2008) 021, [0709.0295].

[46] P. Creminelli and M. Zaldarriaga, Single field consistency relation for the 3-point function, JCAP 0410 (2004) 006, [astro-ph/0407059].

[47] X. Chen, M.-x. Huang, S. Kachru, and G. Shiu, Observational signatures and non-Gaussianities of general single field inflation, JCAP 0701 (2007) 002, [hep-th/0605045].

[48] WMAP Collaboration, E. Komatsu et al., Five-Year Wilkinson Microwave Anisotropy Probe (WMAP) Observations: Cosmological Interpretation, 0803.0547. 